Article

\title{
Competing in Several Areas Simultaneously: The Case of Strategic Asset Markets
}

\author{
Manfred Nermuth \\ Department of Economics, University of Vienna, Hohenstaufengasse 9, A-1010 Vienna, Austria; \\ E-Mail: manfred.nermuth@univie.ac.at; Tel.: +43 1427737440 ; Fax: +43 142779374
}

Received: 20 December 2010; in revised form: 16 March 2011 / Accepted: 7 April 2011 /

Published: 12 April 2011

\begin{abstract}
We characterize the structure of Nash equilibria for a certain class of asset market games. In equilibrium, different assets have different returns, and (risk neutral) investors with different wealth hold portfolios with different structures. In equilibrium, an asset's return is inversely related to the elasticity of its supply. The larger an investor, the more diversified is his portfolio. Smaller investors do not hold all the assets, but achieve higher percentage returns. More generally, our results can be applied also to other "multi-market games" in which several players compete in several arenas simultaneously, like multi-market Cournot oligopolies, or multiple rent-seeking games.
\end{abstract}

Keywords: asset markets; Nash Equilibrium; multigames

\section{Introduction}

\subsection{Motivation}

There are many situations in which a number of players play several different games (interact in different markets) simultaneously, and what a player can do in one game is constrained by what he does in the others (so that the games cannot be analyzed separately). Typically, there is a resource constraint: Each player has a limited amount of some resource (time, money, effort, capacity, ... ) and must decide how much of it to commit to the various "markets" in order to maximize his overall payoff.

Examples include: Strategic investors (with limited budget) in certain asset markets (the subject of this paper), multimarket Cournot oligopoly (with limited capacity), but also political contests where several candidates (with limited time) compete in several constituencies, multiple rent-seeking, etc. 
We study such a situation of "multimarket interaction" for the special case of asset market games of the type considered in [1-3] ${ }^{1}$. These works in turn build on older work on market games of the kind introduced in [6], and especially on work on strategic financial markets, like [7].

The present study was directly motivated by the model in [1]: Here investors decide how to allocate their funds over a number of different assets, which are in constant (unit) supply. Prices are set so as to equate demand and supply. [1] noted that the unique Nash equilibrium of the game coincides with the unique evolutionarily stable strategy (ESS), and also coincides with the "competitive outcome". While a relationship between evolutionary stability and competitive outcomes has also been observed in other contexts $([8,9])$, and thus is not too surprising, the coincidence of competitive outcomes and Nash equilibria is rather striking. It seems that the familiar tension (e.g., in Cournot oligopoly) between price-taking and strategic behavior is absent from these asset markets ${ }^{2}$. It was this observation which originally motivated the present study.

It turns out that a slight modification of the model suffices to remove this anomaly: Rather than constant (inelastic) asset supply, we allow elastic supply, such that different assets may have different elasticities. This recognizes the fact that in an asset market usually only a few investors (e.g., very large, professionally managed funds) will act strategically, that is, be aware that their transactions may influence prices, and take this influence into account for their investment decisions. Only these large, strategic investors will act as players in our market game. Even if the total supply of an asset is really constant, part of it will usually be held by other, nonstrategic market participants, and these may be more or less willing to sell, depending on the price. We model these traders in the simplest possible way by assuming that their aggregate behavior is described by an upward-sloping (possibly constant) supply function (one for each asset). Given this, Nash and competitive outcomes no longer coincide (see Section 1.2).

We also assume that the markets for the various assets are sufficiently separated, so that the supply of each asset depends only on its own price. While such an assumption is certainly restrictive ${ }^{3}$, this permits us to obtain very detailed information about the structure of equilibrium, which would probably be impossible otherwise.

We note also that uncertainty plays no formal role in our analysis. Following part of the literature which motivated this study, we assume that the players are risk neutral, so that only expected payoffs matter. Thus the results in Sections 1-4 of [1] remain true even with elastic supply, as long as all elasticities are the same (Theorem 4.2).

\footnotetext{
${ }^{1}$ This literature was motivated mainly by the question which types of investment strategies will survive in the long run in a stochastic environment. Our focus is different: the structure of equilibrium in the static game.

${ }^{2}$ The salient point is the coincidence of Nash and Walras independently of the number of players. This is quite different from the well-known observation (at least since Cournot) that Nash equilibria tend to Walrasian allocations in the limit, when the number of players goes to infinity, $c f$. [4] and the references given there.

${ }^{3}$ For example, it would not be appropriate for the model of [5]. This model has constant supply of all assets, but features explicitly both strategic and non-strategic (competitive) traders. The non-strategic traders optimize portfolios consisting of the same assets as the strategic traders, the only difference being that they take prices as given. Clearly, the non-strategic traders' total demand for an asset depends on all prices in an essential way, and so does the remaining "supply" left for the strategic traders.
} 
Remark: Even though our analysis was originally motivated by a financial markets literature, such markets are probably not the best examples for our theory. Indeed, in financial markets an important role is played by factors which are not modeled in the present paper, like differential information, risk aversion, short selling, interdependence of asset prices, etc. Combining such features with strategic behavior is quite difficult and requires a different sort of analysis $(c f .[5,7])$.

Instead of financial asset markets, it might be better to think of real assets. For example, the players could be a group of large investment funds buying real estate in various cities ("markets"), like New York, London, Hongkong, ... Each player has a certain budget and must decide how much to invest in the various cities; moreover the players realize that their investments may influence the prices. Alternatively ${ }^{4}$ the players might be large strategic traders who compete in buying various input factors and sell them on the world market, at a given world market price.

\subsection{Summary of Results}

Given the exogenous supply functions (one for each asset) the strategic players decide how much of their available funds to invest in the various assets. Prices are then set to equate supply and demand in each market, exactly as in the classical market games with fixed supply. But note that the game is no longer constant-sum.

The main contribution of the present paper is a detailed characterization of the structure of the possible Nash equilibria of this asset market game, for the non-symmetric case. The results can be summarized as follows: In the symmetric case, when all supply functions have the same elasticity, then there exists a unique Nash equilibrium. It is symmetric and coincides with both the "competitive" and the ESS outcomes (here "competitive" is of course to be understood as a situation in which every investor maximizes profits, taking prices-not supply functions - as given). In this equilibrium, prices are proportional to expected payoffs (prices are "fair", or "correspond to fundamentals") and all investors achieve the same rate of return on their capital. This includes the constant supply situation considered in the literature quoted above. Intuitively, when the supply of all assets is constant, the market game is constant-sum, and a player can increase his payoff only at the expense of the others; therefore the maximization of absolute payoffs (Nash) coincides with the maximization of relative payoffs (ESS) ${ }^{5}$. We show that this continues to hold for variable supply, as long as the supply conditions for all assets are essentially the same (Theorem 4.2).

But the point of the paper is the non-symmetric case where the supply conditions for different assets are genuinely different. Then Nash equilibria are not symmetric, and neither ESS nor competitive. Prices are not fair, and different assets have different return rates. More precisely, the lower the elasticity of supply of an asset, the higher its return. Larger investors are more diversified, with the largest investor holding positive quantities of all assets, but smaller investors buy only some of the assets (the smaller, the fewer). Moreover, larger investors necessarily hold relatively more low-yielding assets and achieve lower average rates of return at equilibrium than small investors (Theorem 4.1); this may be termed the "curse of size".

\footnotetext{
${ }^{4}$ This interpretation was suggested by a referee.

5 This can be made precise, see [10].
} 
These somewhat counterintuitive deviations from the competitive outcome, i.e. different rates of return across assets and/or investors, have nothing to do with market imperfections or other reasons like different degrees of risk aversion etc., but come from the heterogeneity of supply, combined with the strategic interplay among large and small investors: at equilibrium, every investor equalizes the marginal, not the average, rates of return of all assets which he holds in positive quantity. Since marginal rates differ from average ones, and also across investors due to their different wealth, we obtain these heterogeneous portfolios.

Equilibrium is unique in the "symmetric" cases (symmetry w.r.t. assets and/or investors, Theorem 4.2 and Theorem 4.3); in general, we can only prove that there exists at most one Nash equilibrium at which all investors hold all assets (Proposition 4.2; there may exist no such equilibrium).

We also consider competitive and ESS outcomes separately. There always exists a unique ESS, and a unique competitive rate of return (the same for all assets). At the ESS, all investors achieve exactly the competitive rate ${ }^{6}$. At any Nash equilibrium that is not ESS, they achieve strictly more (Theorem 5.1, Theorem 4.4).

The paper is organized as follows. Section 2 introduces the basic model, in Section 3 we study competitive allocations and prove the existence of Nash equilibrium, Section 4 contains the main results, and in Section 5 we study evolutionarily stable strategies. Most proofs, except very short ones, are in the appendix.

\section{Notation and Definitions}

We consider an asset market of the kind studied in [1-3]. There are $i=1,2, \ldots, N$ risk-neutral investors $(N \geq 2)$, and $k=1,2, \ldots, K$ assets $(K \geq 2)$. The initial endowment (with money) of investor $i$ is $W^{i}>0$, and the total money endowment is $W:=\sum_{i} W^{i}$. The (expected) monetary payoff per unit of asset $k$ is $E_{k}>0$, and $S_{k}\left(p_{k}\right)$ is the supply function for asset $k$, where $p_{k} \geq 0$ denotes the price (per unit) of asset $k$. We allow arbitrary supply functions, subject only to the condition that the price elasticity of supply be non-increasing. We denote the supply elasticity of asset $k$ by

$$
\eta_{k}=H_{k}\left(p_{k}\right)=\frac{p_{k} S_{k}^{\prime}\left(p_{k}\right)}{S_{k}\left(p_{k}\right)} \text { for } \quad p_{k}>0
$$

and assume, for $\forall k$ :

S.1. The supply function $S_{k}\left(p_{k}\right)$ is continuous and nondecreasing for $p_{k} \geq 0$, and strictly positive for $p_{k}>0$.

S.2. The supply function $S_{k}\left(p_{k}\right)$ is twice continuously differentiable (possibly with infinite slope at $\left.p_{k}=0\right)^{7}$.

S.3. The elasticity $H_{k}\left(p_{k}\right)$ is non-increasing on $(0, \infty)$.

Remark: It may be that $S_{k}(0)=0$ or $S_{k}(0)>0$; we will show (cf. Lemma A.2 in the appendix) that the latter case occurs if and only if supply is constant, $S_{k}\left(p_{k}\right)=\bar{S}_{k}>0 \quad \forall p_{k} \geq 0$. This is the situation

\footnotetext{
${ }^{6}$ A connection between evolutionary stability and competitive outcomes has also been found in other contexts ([9]).

${ }^{7}$ For a precise statement see $\mathbf{S . 4}$ in the appendix.
} 
considered in the literature quoted above ${ }^{8}$. To justify a variable supply in the strategic $N$-player game among "big" investors which we are going to study, we may imagine that the asset is also held by a large number of small, nonstrategic market participants. These traders are simply willing to sell more of an asset if its price goes up; their aggregate behavior is captured by the supply functions $S_{k}$.

Each investor $i$ invests his whole wealth $W^{i}$ in the $K$ available assets, i.e. he chooses a vector $w^{i}=\left(w_{1}^{i}, \ldots, w_{K}^{i}\right)$ in his budget set

$$
B^{i}=B^{i}\left(W^{i}\right)=\left\{w^{i} \in \mathbb{R}_{+}^{K} \mid \sum_{k} w_{k}^{i}=W^{i}\right\}
$$

where $w_{k}^{i} \geq 0$ is the amount of money invested in asset $k$ by investor $i$.

The set $B^{i}$ is $i$ 's strategy space; it is a nonempty, compact and convex subset of $\mathbb{R}_{+}^{K}$. The joint strategy space is $B:=\prod_{i} B^{i} \subset \mathbb{R}_{+}^{K N}$. A strategy profile is denoted by $w=\left(w^{1}, \ldots w^{N}\right) \in B$. Given $w$, we write $w_{k}:=\sum_{i} w_{k}^{i}$ for the total amount of money invested in asset $k$ by all investors $\left(w^{i}\right.$ is a vector, but $w_{k}$ is a scalar!). If $w_{k}>0$ we say that market $k$ is active (at $w$ ).

Alternatively (and equivalently), the behavior of an investor $i$ can be described in percentage terms, i.e. by $\alpha^{i}=\left(\alpha_{1}^{i}, \ldots \alpha_{K}^{i}\right)$, where $\alpha_{k}^{i}=w_{k}^{i} / W^{i}$ denotes the fraction of investor $i$ 's wealth invested in asset $k$. This formulation makes the game appear more symmetric and is useful in certain contexts (e.g., to study evolutionary stability, see Section 5). The vector $\alpha^{i}$ describes how investor $i$ allocates his money among the various assets (30\% in German real estate, $3 \%$ in Gold, etc.); for convenience we refer to $\alpha^{i}$ also as investor $i$ 's portfolio. Similarly, we use the word "market portfolio" for the vector $\left(w_{1} / W, \ldots w_{N} / W\right)$, describing the percentage allocation of total wealth $W$ over the $K$ asset types (in the finance literature, the term "market portfolio" has a slightly different meaning, but this should cause no confusion).

For $p_{k}>0$, we denote by $r_{k}:=E_{k} / p_{k}$ the (gross) rate of return per dollar of asset $k$. In a competitive equilibrium, with price-taking risk-neutral investors, $r_{k}$ must be the same for all assets ( $c f$. Section 3.1).

REMARK: even though, in the game to be considered below, investors are constrained to choose $w^{i} \in B^{i}$, many of the following considerations do not depend on this restriction, but are valid for arbitrary nonnegative $w^{i} \in \mathbb{R}^{K}$. We will therefore, whenever appropriate, pay no attention to the budget constraints and consider arbitrary strategy profiles $w \in \mathbb{R}_{+}^{K N}$.

Given $w \in \mathbb{R}_{+}^{K N}$, the prices $p_{k}$ are determined so as to clear markets, i.e. such that $\forall k$

$$
p_{k} S_{k}\left(p_{k}\right)=w_{k}
$$

It is easy to see ( $c f$. Lemmas A.1, A.3) that this defines a unique price $p_{k}=P_{k}\left(w_{k}\right)$ for every $w_{k} \geq 0$; and that the price function $p_{k}=P_{k}\left(w_{k}\right)$ is differentiable and strictly increasing, with $P_{k}(0)=0$, $\lim _{w_{k} \rightarrow \infty} P_{k}\left(w_{k}\right)=\infty$. Therefore the rate of return,

$$
r_{k}=R_{k}\left(w_{k}\right):=\frac{E_{k}}{P_{k}\left(w_{k}\right)}=\frac{E_{k} S_{k}\left(P_{k}\left(w_{k}\right)\right)}{w_{k}}
$$

\footnotetext{
${ }^{8}$ Note on normalization: in the literature with fixed supply, it is frequently assumed (w.l.o.g.) that the total supply of each asset is equal to unity: $\bar{S}_{k}=1 \forall k$. In our context, a possible normalization is to choose units such that the return per unit of each asset is equal to unity: $E_{k}=1 \quad \forall k$. This is sometimes useful, e.g., in the context of Theorem 4.2.
} 
is also a differentiable function of $w_{k}$, and strictly decreasing in $w_{k}$, with $\lim _{w_{k} \rightarrow 0} R_{k}\left(w_{k}\right)=\infty$ and $\lim _{w_{k} \rightarrow \infty} R_{k}\left(w_{k}\right)=0$. Note in particular that if the total investment in asset $k$ goes to zero, $w_{k} \rightarrow 0$, then the expected rate of return $R_{k}=R_{k}\left(w_{k}\right)$ becomes arbitrarily large. This will ensure that in Nash equilibrium all markets are active.

We conclude this section with a formula that will be useful in the sequel. Note that by definition (2), $P_{k}\left(w_{k}\right) S_{k}\left(P_{k}\left(w_{k}\right)\right) \equiv w_{k}$. Totally differentiating gives:

$$
P_{k}^{\prime}\left(w_{k}\right) S_{k}\left(P_{k}\left(w_{k}\right)\right)+P_{k}\left(w_{k}\right) S_{k}^{\prime}\left(P_{k}\left(w_{k}\right)\right) P_{k}^{\prime}\left(w_{k}\right) \equiv 1 \quad \Leftrightarrow \quad P_{k}^{\prime} S_{k}\left[1+\eta_{k}\right] \equiv 1
$$

Using $S_{k}=w_{k} / P_{k}$ we obtain:

$$
0<\frac{1}{1+H_{k}\left[P_{k}\left(w_{k}\right)\right]} \equiv \frac{P_{k}^{\prime}\left(w_{k}\right) w_{k}}{P_{k}\left(w_{k}\right)} \leq 1
$$

Expression (4) is the elasticity of the price $p_{k}=P_{k}\left(w_{k}\right)$ with respect to the total amount of money $w_{k}$ invested in asset $k$. It lies between zero and one because $\eta_{k} \geq 0$ by $\mathbf{S . 1}$ and is equal to one if and only if the supply elasticity $\eta_{k}=H_{k}\left[P_{k}\left(w_{k}\right)\right]$ is zero (i.e., supply is constant).

\section{The Market Game}

Given a strategy profile $w \in \mathbb{R}_{+}^{K N}$, the amount of asset $k$ allocated to investor $i$ is given by:

$$
x_{k}^{i}(w):= \begin{cases}w_{k}^{i} / P_{k}\left(w_{k}\right) & \text { if } w_{k}>0 \quad\left(\Rightarrow p_{k}>0\right) \\ 0 & \text { if } w_{k}=0 \text { (market } k \text { is not active) }\end{cases}
$$

If market $k$ is active we may also write

$$
x_{k}^{i}(w)=\frac{w_{k}^{i}}{w_{k}} S_{k}\left(P_{k}\left(w_{k}\right)\right) \quad\left(w_{k}>0\right)
$$

If $i$ is the only investor who holds asset $k\left(w_{k}^{i}=w_{k}\right)$, then

$$
x_{k}^{i}(w)= \begin{cases}S_{k}\left(P_{k}\left(w_{k}\right)\right) & \text { if } w_{k}^{i}>0 \text { or } S_{k}(0)=0 \\ 0 \quad\left[\neq S_{k}(0)\right] & \text { if } w_{k}^{i}=0 \text { and } S_{k}(0) \neq 0\end{cases}
$$

with a discontinuity at $w_{k}^{i}=0$ if $S_{k}(0)>0$. The payoff of investor $i$ is then given by

$$
\pi^{i}(w)=\sum_{k} E_{k} x_{k}^{i}(w)=\sum_{\substack{k \\ w_{k}>0}} w_{k}^{i} R_{k}\left(w_{k}\right)
$$

These data define the asset market game $G$ among the $N$ investors, with strategy spaces $B^{i}$ and payoff functions $\pi^{i}$.

In this formulation the strategies and payoffs of large and small investors are not directly comparable, but we can make them so by expressing everything in percentage terms, i.e. by dividing both the invested amounts $w_{k}^{i}$ and the payoff $\pi^{i}$ of an investor $i$ by his initial wealth $W^{i}$. This gives a strategically equivalent game $\bar{G}$ as follows. The strategy of investor $i$ is his portfolio $\alpha^{i}=\left(\alpha_{1}^{i}, \ldots, \alpha_{K}^{i}\right)=(W)^{-1}$. 
$\left(w_{1}^{i}, \ldots, w_{K}^{i}\right)$, his strategy space is the $(K-1)$-dimensional unit simplex $\Delta^{K}$, a joint strategy is written $\alpha=\left(\alpha^{1}, \ldots, \alpha^{N}\right)$, and the payoff of investor $i$ in the modified game $\bar{G}$ is his return per dollar

$$
r^{i}=\bar{\pi}^{i}(\alpha)=\sum_{\substack{k \\ \alpha_{k}^{i}>0}} \alpha_{k}^{i} \bar{R}_{k}(\alpha)=\frac{\pi^{i}(w)}{W^{i}}
$$

where $\bar{R}_{k}(\alpha):=R_{k}\left(\sum_{i} W^{i} \alpha_{k}^{i}\right)$; and $\sum_{i} W^{i} \alpha_{k}^{i}=\sum_{i} w_{k}^{i}=w_{k}$ is the total amount invested in asset $k$ if the investors use the joint strategy $\alpha$.

This formulation of the game is quite natural. Indeed, investors frequently describe their strategy by stating how many percent of their total funds they invest in various types of assets, and they describe their payoffs by stating the percentage return on their portfolio (e.g., $4.5 \%$ p.a.), not the absolute quantities. We will use the two equivalent formulations $G$ and $\bar{G}$ interchangeably, according to convenience, and refer to both $G$ and $\bar{G}$ as the "asset market game".

Our main interest will be in determining the structure of the Nash equilibria of this game, corresponding to strategic (fully rational) behavior of the agents (Section 4). But we will also consider other solution concepts: Competitive outcomes corresponding to price-taking behavior, and evolutionarily stable strategies (ESS) in the sense of [11], which are motivated by certain types of boundedly rational behavior (imitation) (Section 5). The relationships between these solution concepts will also be clarified. Moreover, it is easy to see that the game $G$ is constant-sum if and only if all supply functions $S_{k}$ are constant (see Lemma A.4 in the appendix).

Given a strategy profile $w \in B$, the expected payoff from asset k, $E_{k} S_{k}=E_{k} S_{k}\left[P_{k}\left(w_{k}\right)\right]$, and the total expected payoff, $E(w):=\sum_{k} E_{k} S_{k}$, are determined. We say that investor $i$ follows the proportional investment rule if the amount invested by him in each asset $k$ is proportional to the expected payoff $E_{k} S_{k}$ of this asset, i.e. if there is $\gamma^{i}>0$ such that $w_{k}^{i}=\gamma^{i} E_{k} S_{k} \forall k$ (or $\alpha_{k}^{i}=E_{k} S_{k} / E(w)$; "investing according to the fundamentals").

\subsection{Competitive Allocations}

A profile $w=\left(w^{1}, \ldots w^{N}\right)$ (resp. the corresponding asset allocation) is called competitive if all assets have the same rate of return, i.e. if there exists $\hat{r}$ such that for all $k$

$$
R_{k}\left(w_{k}\right)=\hat{r}
$$

In this case $p_{k}=\frac{1}{\hat{r}} E_{k} \forall k$, i.e. prices are proportional to expected payoffs. We also say that prices are fair. Clearly, in a competitive allocation, the common rate of return $\hat{r}$ is equal to the total payoff divided by the total initial money endowment

$$
\hat{r}=\frac{E(w)}{W}
$$

(to see this, use (3) to write $w_{k}=\hat{r}^{-1} E_{k} S_{k}$ and sum over $k$ ).

Lemma 3.1. There exists a unique competitive rate of return $\hat{r}>0$. It depends only on the total money endowment $W$, but not on the distribution of wealth $W^{1}, \ldots W^{N}$.

Proof: For $r>0$, define $w_{k}(r)>0$ by $R_{k}\left[w_{k}(r)\right]=r$. The properties of the return function $R_{k}(\cdot)$ imply that $w_{k}(r)$ is well defined and strictly decreasing in $r$ (from $\infty$ to 0 ); hence there exists a unique $\hat{r}>0$ such that $\sum_{k} w_{k}(\hat{r})=W$. 
Define $\hat{w}_{k}$ by $R_{k}\left(\hat{w}_{k}\right)=\hat{r}$. In competitive equilibrium the return rate $\hat{r}$, the prices $\hat{p}_{k}=E_{k} / \hat{r}$, the profits $\pi^{i}(w)=\sum_{k} w_{k}^{i} R_{k}=W^{i} \hat{r}$, and the amounts $\hat{w}_{k}$ are uniquely determined, but not the asset allocation. Indeed, from the viewpoint of a price-taking investor $i$, any strategy $w^{i}=\left(w_{1}^{i}, \ldots w_{K}^{i}\right) \in B^{i}$ is profit maximizing, since all assets yield the same return. Thus there are infinitely many competitive allocations, characterized by the condition that the total amounts $w_{k}=\sum_{i}^{N} w_{k}^{i}$ invested in the various assets satisfy $w_{k}=\hat{w}_{k}$. Among these, a special role is played by the proportional investment rule.

Lemma 3.2. (i) There exists a unique profile $\hat{w} \in B$ in which all investors follow the proportional investment rule. This profile is competitive and is given by $\hat{w}_{k}^{i}=\hat{w}_{k} \frac{W^{i}}{W}$, where $\hat{w}_{k}$ is given by $R_{k}\left(\hat{w}_{k}\right)=$ $\hat{r}$. (ii) Let $w \in B$ be a competitive profile such that all investors hold the same portfolio $\alpha^{i}=\alpha^{j} \forall i, j$. Then $w=\hat{w}$ and $\alpha^{i}=\hat{\alpha} \forall i$, where $\hat{\alpha}=\left(\hat{\alpha}_{1}, \ldots \hat{\alpha}_{K}\right):=\frac{1}{W}\left(\hat{w}_{1}, \ldots \hat{w}_{K}\right) \in \Delta^{K}$ is the market portfolio corresponding to the profile $\hat{w} \in \mathbb{R}$.

Let us call a profile $w=\left(w^{1}, \ldots w^{N}\right) \in B$ symmetric ${ }^{9}$ if all investors hold the same portfolio, i.e. if $\alpha^{i}=\alpha^{j}$ for all $i, j$, where $\alpha^{i}=\left(1 / W^{i}\right) w^{i}$. Lemma 3.2 (ii) says that $\hat{w}$ is the only symmetric competitive profile. We shall see below that this "proportional competitive profile" $\hat{w}$ has certain special properties. In particular, if a Nash equilibrium allocation $w$ is competitive (this is not the case in general), then it coincides with $\hat{w}$ (Propostion 4.1). Moreover, $\hat{w}$ is the unique profile that is evolutionarily stable in the sense of [11] (Theorem 5.1).

\subsection{Nash Equilibrium}

A profile $w^{*}=\left(w^{* 1}, \ldots w^{* N}\right) \in B$ is a Nash equilibrium of the game $G$ if for all investors $i=1, \ldots N$

$$
\pi^{i}\left(w^{*}\right) \geq \pi^{i}\left(w^{i}, w^{*-i}\right) \quad \forall w^{i} \in B^{i}
$$

where $\left(w^{i}, w^{*-i}\right)$ denotes the profile $w^{*}$ with $i$ 's strategy $w^{* i}$ replaced by $w^{i}$.

Theorem 3.1. (i) The asset market game $G=G\left[\left(W^{i}\right),\left(E_{k}, S_{k}\right)\right]$ has a Nash equilibrium. (ii) At any equilibrium, all markets are active. (iii) Every equilibrium is strict.

The proof of the theorem is essentially routine, based on the observation that the payoff functions are concave. Some care must be taken because of possible discontinuities at the boundary of the budget sets. Details are in the appendix.

The results on the structure of equilibrium in the next section are based on the following observation. The marginal return to investor $i$ from asset $k$ can be written as

$$
\frac{\partial \pi_{k}^{i}(w)}{\partial w_{k}^{i}}=r_{k}\left[1-\frac{w_{k}^{i}}{w_{k}} \frac{1}{1+\eta_{k}}\right] \text { for } w_{k}>0
$$

where $r_{k}=R_{k}\left(w_{k}\right), \eta_{k}=H_{k}\left[P_{k}\left(w_{k}\right)\right]$. Indeed, by definition, $x_{k}^{i}(w)=w_{k}^{i} / P_{k}\left(w_{k}\right)$, and by (4), $1 / 1+\eta_{k}=P_{k}^{\prime} w_{k} / P_{k}$, therefore

$$
\frac{\partial x_{k}^{i}(w)}{\partial w_{k}^{i}}=\frac{P_{k}-w_{k}^{i} P_{k}^{\prime}}{\left(P_{k}\right)^{2}}=\frac{1}{P_{k}}\left[1-\frac{w_{k}^{i}}{w_{k}} \frac{w_{k} P_{k}^{\prime}}{P_{k}}\right]=\frac{1}{P_{k}}\left[1-\frac{w_{k}^{i}}{w_{k}} \frac{1}{1+\eta_{k}}\right]
$$

${ }^{9}$ The game $G$ (or $\bar{G}$ ) is not symmetric, see Section 5 . 
Formula (12) follows immediately from the definitions (8) and $r_{k}=E_{k} / P_{k}$. The "Nash term" $-w_{k}^{i} / w_{k}\left(1+\eta_{k}\right)$ in (12) reflects the fact that an increase of $w_{k}^{i}$ reduces the return rate of asset $k$; it disappears only under the "competitive" assumption of infinitely elastic supply $\left(\eta_{k}=\infty\right)$.

\section{Structure of Nash Equilibrium}

This section contains the main results. Consider an equilibrium $w=\left(w^{1}, \ldots w^{N}\right)$ of $G$ with associated prices $p_{k}=P_{k}\left(w_{k}\right)$, supplies $S_{k}\left(p_{k}\right)$, asset returns $r_{k}=R_{k}\left(w_{k}\right)=E_{k} / p_{k}$, and elasticities $\eta_{k}=H_{k}\left(p_{k}\right)$. Denote by $E=E(w)=\sum_{k} E_{k} S_{k}\left(p_{k}\right)$ the aggregate payoff in the economy, and let $R=E(w) / W$ be the aggregate rate of return (remember that $W=\sum_{i} W^{i}$ is the aggregate initial wealth). If $w_{k}^{i}>0$ we say that investor $i$ holds asset $k$, or that he is active in market $k$. Denote further by $r^{i}:=\bar{\pi}^{i}(\alpha)=\pi^{i}(w) / W^{i}$ the rate of return investor $i$ gets on his capital, and write $\alpha^{i}=\left(\alpha_{1}^{i}, \ldots \alpha_{K}^{i}\right)$, where $\alpha_{k}^{i}=w_{k}^{i} / W^{i}$, for the portfolio associated with $w^{i}$.

Theorem 4.1. Let $w=\left(w_{k}^{i}\right)$ be an equilibrium, with investors and assets ordered such that $W^{1} \geq W^{2} \geq$ $\cdots \geq W^{N}$ and $r_{1} \leq r_{2} \leq \cdots \leq r_{K}$. Then

1. the largest investor $(i=1)$ holds all assets: $w_{k}^{1}>0 \quad \forall k$,

2. the asset with the highest return $(k=K)$ is held by every investor: $w_{K}^{i}>0 \quad \forall i$,

3. if investor $i$ holds asset $k\left(w_{k}^{i}>0\right)$, then:

(a) $i$ also holds all assets with higher or equal returns $\left(w_{\ell}^{i}>0\right.$ for $\left.r_{\ell} \geq r_{k}\right)$

(b) all larger investors $j \leq i$ also hold at least the same quantity of asset $k\left(w_{k}^{j} \geq w_{k}^{i}\right)$, with strict inequality iff $j$ is strictly larger than $i\left(W^{j}>W^{i}\right)$.

4. larger investors hold relatively more low-yielding assets in the following sense: whenever $W^{i} \geq W^{j}$, then the portfolios $\alpha^{i}$, $\alpha^{j}$ satisfy $\quad \alpha_{1}^{i}+\alpha_{2}^{i}+\ldots \alpha_{k}^{i} \geq \alpha_{1}^{j}+\alpha_{2}^{j}+\ldots \alpha_{k}^{j} \quad \forall k$

5. the lower the elasticity of supply for an asset, the higher its return:

$$
r_{k}<r_{\ell} \Leftrightarrow \eta_{k}>\eta_{\ell} \quad \text { and } \quad r_{k}=r_{\ell} \quad \Leftrightarrow \quad \eta_{k}=\eta_{\ell}
$$

6. larger investors have lower return rates: $r^{i} \geq r^{j} \Leftrightarrow W^{i} \leq W^{j}$

Let $w$ be an equilibrium, and denote by $\lambda^{i}$ the Lagrange multiplier associated with investor $i$ 's budget constraint. By formula (12), the following first-order conditions [FOC] must hold, for $i=1, \ldots N$ :

$$
\frac{\partial \pi^{i}(w)}{\partial w_{k}^{i}}= \begin{cases}r_{k}\left[1-\frac{w_{k}^{i}}{w_{k}} \frac{1}{1+\eta_{k}}\right] & =\lambda^{i} \quad \forall k \text { with } w_{k}^{i}>0 \\ r_{k} & \leq \lambda^{i} \quad \forall k \text { with } w_{k}^{i}=0\end{cases}
$$

The proof of the various assertions in the Theorem is based on a careful examination of these first-order conditions. Details are in the appendix.

Table 1 summarizes the results of Theorem 4.1. At a Nash equilibrium, in general, different assets have different returns; and not every investor is active in all markets. Larger investors are active in 
more markets. The more elastic the supply of an asset, the lower its return rate at equilibrium. Larger investors hold relatively more low-yielding assets, and achieve lower average rates of return on their capital. Investors with the same wealth use the same strategy.

Table 1. The structure of equilibrium.

\begin{tabular}{|c|c|c|c|c|c|c|c|c|c|c|c|c|c|}
\hline & $w_{k}^{i}$ & $\begin{array}{l}\eta_{1} \\
r_{1}\end{array}$ & & $\begin{array}{l}\eta_{2} \\
r_{2}\end{array}$ & $\begin{array}{l}\geq \\
\leq\end{array}$ & $\begin{array}{l}\cdots \\
\ldots\end{array}$ & $\begin{array}{l}\geq \\
\leq\end{array}$ & $\begin{array}{l}\eta_{k} \\
r_{k}\end{array}$ & $\begin{array}{l}\geq \\
\leq\end{array}$ & $\begin{array}{l}\cdots \\
\ldots\end{array}$ & $\begin{array}{l}\geq \\
\leq\end{array}$ & $\begin{array}{l}\eta_{K} \\
r_{K}\end{array}$ & $\begin{array}{l}\text { row } \\
\text { sums }\end{array}$ \\
\hline$r^{1}$ & $\lambda^{1}$ & $w_{1}^{1}$ & & $w_{2}^{1}$ & & $\ldots$ & & $w_{k}^{1}$ & & & & $w_{K}^{1}$ & $W^{1}$ \\
\hline$\leq$ & $\leq$ & & & & & & & $\geq$ & & & & $\geq$ & $\geq$ \\
\hline$r^{2}$ & $\lambda^{2}$ & 0 & & & $w_{k^{2}}^{2}$ & $\ldots$ & & $w_{k}^{2}$ & & & & $w_{K}^{2}$ & $W^{2}$ \\
\hline$\leq$ & $\leq$ & & & & & & & $\geq$ & & & & $\geq$ & $\geq$ \\
\hline$\cdots$ & $\ldots$ & & & & & $\ldots$ & & & & $\ldots$ & & & $\ldots$ \\
\hline$r^{i}$ & $\lambda^{i}$ & 0 & & 0 & & $\ldots$ & & $w_{k^{i}}^{i}$ & & $\ldots$ & & $w_{K}^{i}$ & $W^{i}$ \\
\hline$\leq$ & $\leq$ & & & & & & & & & $\ldots$ & & $\geq$ & $\geq$ \\
\hline$\ldots$ & $\ldots$ & & & & & $\ldots$ & & & & $\ldots$ & & & $\ldots$ \\
\hline$r^{N}$ & $\lambda^{N}$ & 0 & & 0 & & $\ldots$ & & 0 & & $w_{k^{N}}^{N}$ & & $w_{K}^{N}$ & $W^{N}$ \\
\hline & $\begin{array}{c}\text { column } \\
\text { sums }\end{array}$ & $w_{1}$ & & $w_{2}$ & & $\ldots$ & & $w_{k}$ & & & & $w^{K}$ & $W$ \\
\hline
\end{tabular}

An equilibrium allocation: rows correspond to investors and columns to assets. $w_{k}^{i}$ is the amount invested in asset $k$ by investor $i$. If $W^{i}=W^{j}$, then the corresponding rows are identical; if $W^{i}>W^{j}$, then $r^{i} \leq r^{j}, \lambda^{i}<\lambda^{j}$, and $w_{k}^{i}>w_{k}^{j}$, except when $w_{k}^{i}=0$. For any two adjacent columns $k$ and $k+1, r_{k}=r_{k+1}$ iff $\eta_{k}=\eta_{k+1}$. In this case, $w_{k}^{i}>0 \Rightarrow w_{k+1}^{i}>0 \forall i$.

Intuitively, $r_{k}$ is the average return of asset $k$, and by (12),

$$
\frac{\partial \pi^{i}(w)}{\partial w_{k}^{i}}=r_{k}\left[1-\frac{w_{k}^{i}}{w_{k}} \frac{1}{1+\eta_{k}}\right]
$$

is the marginal return of asset $k$ for investor $i$. The marginal return is always less than the average return $r_{k}$ (because an extra dollar invested in an asset also pushes up its price), but it gets closer to $r_{k}$ when the elasticity $\eta_{k}$ increases. Since marginal, not average, returns must be equal at equilibrium, we get the inverse relationship between $r_{k}$ and $\eta_{k}$ asserted in the Theorem. Moreover, the discrepancy between marginal and average return increases with $w_{k}^{i}$, i.e. it is larger for larger investors

Thus with variable supply, Nash equilibrium allocations are not competitive in general (prices are not fair). Example 1 illustrates such a case. This deviation of asset prices from the expected return has nothing to do with risk aversion of our investors, but results from their strategic interaction in a situation where the supply conditions of different assets differ. Of course, in our model, for any asset $k$, the exogenous supply function $S_{k}\left(p_{k}\right)$ summarizes the aggregate behavior of the (non-strategic) "rest of the 
market". This "rest" may contain risk-averse traders (or even traders with no rational attitude to risk at all). While we do not model these traders explicitly, it may of course be that the elasticity of supply of some asset $k$ depends on its riskiness; and to the extent that this is the case, our equilibrium prices also reflect risk, at least indirectly.

Moreover, we observe what we have termed the "curse of size": larger investors achieve lower average return rates at equilibrium. Again this has nothing to do with any differences in the skills or preferences of investors, but results from the equalization of marginal, not average, return rates at a Nash equilibrium. A typical small investor concentrates his portfolio on the highest-yielding assets, achieving a high average return rate; and because he is small, his marginal return is high, too. A large investor has a much lower marginal return and finds it profit-maximizing to hold also the lower-yielding assets, thus depressing his average return.

Example 1. Let $N=2, K=2, E_{k}=1 \forall k$, and $S_{1}\left(p_{1}\right)=p_{1}, S_{2}\left(p_{2}\right)=1$. Then $\eta_{1}=1, P_{1}\left(w_{1}\right)=\sqrt{w_{1}}$, $R_{1}\left(w_{1}\right)=1 / \sqrt{w_{1}}$ and $\eta_{2}=0, P_{2}\left(w_{2}\right)=w_{2}, R_{2}\left(w_{2}\right)=1 /\left(w_{2}\right)$. Assume that the initial endowments are $W^{1}=4.75, W^{2}=0.25$, so that $W=5$. Then the unique Nash equilibrium is given in Table 2. It is easy to check that the first-order conditions are satisfied, with $r^{1}>\lambda^{2}$, i.e. investor $i=2$ does not hold asset $k=1\left(w_{1}^{2}=0\right)$. The total payoff at equilibrium is $E=\pi^{1}+\pi^{2}=3$, the average return is $R=E / W=0.6$, and the competitive rate is $\hat{r}=0.558$.

Table 2. Nash equilibrium in Example 1.

\begin{tabular}{ll|ll|ll} 
& & $\eta_{1}=1$ & $\eta_{2}=0$ & & \\
& & $r_{1}=0.5$ & $r_{2}=1$ & & \\
\hline$r^{1}=.578$ & $\lambda^{1}=0.25$ & $w_{1}^{1}=4$ & $w_{2}^{1}=0.75$ & $W^{1}=4.75$ & $\pi^{1}=2.75$ \\
$r^{2}=1$ & $\lambda^{2}=0.75$ & $w_{1}^{2}=0$ & $w_{2}^{2}=0.25$ & $W^{2}=0.25$ & $\pi^{2}=0.25$ \\
\hline$R=0.6$ & & $w_{1}=4$ & $w_{2}=1$ & $W=5$ & $E=3$
\end{tabular}

Theorem 4.2 below shows that the deviation of Nash equilibrium prices from their fair values is not due to the variability (as opposed to constancy) of supply per se, but to differences in the supply conditions of different assets. As a preliminary step, the following proposition shows that the only competitive profile that can possibly be a Nash equilibrium is the "proportional competitive" profile $\hat{w}$ defined in Lemma 3.2.

Proposition 4.1. Let $w$ be a Nash equilibrium profile. Then $w$ is competitive if and only if $w=\hat{w}$ (i.e., all investors use the proportional investment rule, cf. Lemma 3.2).

Proof: If the equilibrium satisfies $w=\hat{w}$, it is competitive by Lemma 3.2 (i). Conversely, assume that a Nash equilibrium $w$ is competitive. By Lemma 3.1, $r_{k}=\hat{r}, w_{k}=\hat{w}_{k} \forall k$. By Theorem 4.1, all elasticities are equal, $\eta_{k}=\hat{\eta} \forall k$, and every investor $i$ holds all assets. Therefore the first-order condition for an investor $i$ takes the form

$$
\hat{r}\left[1-\frac{w_{k}^{i}}{\hat{w}_{k}} \frac{1}{1+\hat{\eta}}\right]=\lambda^{i} \quad \forall k
$$


Thus, there is $\gamma^{i}>0$ such that $w_{k}^{i} / \hat{w}_{k}=\gamma^{i} \forall k$, and all investors hold the same portfolio in percentage terms, $\alpha^{i}=\alpha^{j}$. By Lemma 3.2 (ii), $w=\hat{w}$. In particular, there is at most one competitive Nash equilibrium. A sufficient condition for the equilibrium to be competitive is given in the next theorem.

Theorem 4.2. Assume that there exists a common elasticity function $H(\cdot)$ such that $H_{k}\left(p_{k}\right)=H\left(p_{k} / E_{k}\right)$ for all assets $k$. Then there exists a unique equilibrium $w$, and $w=\hat{w}$.

The assumption of the Theorem means that all supply functions $S_{k}(\cdot)$ have the same elasticity function, provided units are chosen such that the payoff per unit is the same for all assets. Such a normalization (e.g., $E_{k}=1$ ) is always possible w.l.o.g. ( $c f$. footnote 8). In particular, the assumption of the theorem is satisfied (with $H \equiv 0$ ) if supply is constant.

Proof: We prove that all $r_{k}$ are equal at equilibrium. Let $r_{1} \leq r_{2} \leq \cdots \leq r_{K}$ as in Theorem 4.1. Then $H_{1}\left(p_{1}\right) \geq H_{K}\left(p_{K}\right)$, hence by assumption $H\left(p_{1} / E_{1}\right) \geq H\left(p_{K} / E_{K}\right)$. By S.3, the function $H$ is non-increasing, hence $p_{1} / E_{1} \leq p_{K} / E_{K}$ or $r_{1}=E_{1} / p_{1} \geq E_{K} / p_{K}=r_{K}$. Therefore $r_{k}=\hat{r} \forall k$ and the equilibrium is competitive. By Proposition $4.1, w=\hat{w}$ uniquely.

A competitive equilibrium, if it exists, is symmetric. There may exist non-competitive symmetric equilibria ( $c f$. Theorem 4.3), but a game can have at most one symmetric equilibrium. In fact, more is true: a game can have at most one equilibrium in which all investors are active in all markets:

Proposition 4.2. There is at most one Nash equilibrium in which every investor holds all assets, $w_{k}^{i}>0$ $\forall i, \forall k$.

Since in a symmetric equilibrium every investor must hold all assets, we obtain immediately:

Corollary 4.1. There exists at most one symmetric Nash equilibrium.

Another interesting special case is when all investors have the same wealth, $W^{i}=W^{0} \forall i$ (but supply elasticities may differ).

Theorem 4.3. Assume that all investors have the same wealth, $W^{i}=W^{0}>0 \forall i$. Then there exists a unique equilibrium, and all investors choose the same strategy: $w^{i}=w^{j} \forall i, j$.

Proof: Consider an equilibrium and number investors and assets as in Theorem 4.1. By assumption, all investors have the same wealth, and by monotonicity (45) $w_{k}^{i} \geq w_{k}^{i+1}$. This is only possible if $w_{k}^{i}=w_{k}^{j}$ $\forall i, j, \quad$ i.e., if $w_{k}^{i}=\frac{1}{N} w_{k} \forall i, k$. Thus the equilibrium is symmetric, and by Corollary 4.1, unique.

Remark: If the Nash equilibrium is competitive, then all investors necessarily choose the same portfolio, by Proposition 4.1. The converse is not true: In Theorem 4.3, for example, all investors choose the same portfolio, but assets with constant, but different supply elasticities have different return rates.

Are the investors better off at Nash equilibrium than at a competitive profile? Consider an arbitrary profile $w \in B$ in which all markets are active, so that the return rates $r_{k}=r_{k}\left(w_{k}\right)$ are well defined for all $k$. Then the payoff of investor $i$ can be written $\pi^{i}(w)=\sum_{k} w_{k}^{i} r_{k}$, and his (gross) rate of return (per dollar invested) is

$$
r^{i}=\bar{\pi}^{i}(\alpha)=\sum_{k} \frac{w_{k}^{i}}{W^{i}} r_{k}=\sum_{k} \alpha_{k}^{i} r_{k}
$$


a convex combination of the quantities $r_{1}, \ldots r_{K}$. In a competitive profile, $r_{k}=\hat{r} \forall k$, so that of course $r^{i}=\hat{r}$. If the profile $w$ is not competitive, then some $r_{k}$ must be strictly smaller than $\hat{r}$, and some strictly larger (because the functions $r_{k}=R_{k}\left(w_{k}\right)$ are strictly decreasing, and the sum $\sum_{k} w_{k}=W$ is fixed). Thus it is not clear a priori if an investor's equilibrium rate of return is greater or smaller than $\hat{r}$, especially it is not clear for large investors who hold relatively more low-yielding assets (Theorem 4.1 (4)). In fact, investors never do worse at a Nash equilibrium than at a competitive allocation:

Theorem 4.4. Let $w^{*} \in B$ be a Nash equilibrium that is not a competitive allocation. Then every investor $i$ achieves a strictly higher rate of return than the competitive rate:

$$
r^{i}=\frac{\pi^{i}\left(w^{*}\right)}{W^{i}}>\hat{r} \quad \forall i
$$

\section{Evolutionarily Stable Strategies}

The concept of an evolutionarily stable strategy (ESS) for a finite game introduced by [11] is defined for symmetric games as follows. A strategy $s$ in the common strategy space $S$ is an ESS if, starting from a symmetric situation where everybody uses the strategy $s$, the payoff of a single deviator after deviation is never greater than the payoff of the others (the non-deviators) after this deviation. I.e. no single deviation from the ESS improves the deviator's relative position.

Although this is a static concept, it can sometimes be shown that an ESS is also a stable rest point of some suitably specified "evolutionary" dynamic process of imitation and experimentation ([1]).

Since the game $G$ is not symmetric due to the unequal wealth of different investors, neither the definition of an ESS nor the idea of imitation is directly applicable. But one can argue that these concepts make sense if we think in percentage terms, i.e., in the more symmetric formulation $\bar{G}$. Indeed, in $\bar{G}$, every investor, large or small, has the same strategy space $\Delta^{K}$, and the payoffs of different players can meaningfully be compared. An investor making 3\% with a portfolio of a certain composition might look at some other investor (bigger or smaller) making $4 \%$ with a portfolio of a different composition, and might imitate the composition of the other, seemingly more successful, portfolio. In the spirit of bounded rationality, such imitative behavior is certainly justifiable. If we accept this, it becomes meaningful to define an ESS as a strategy which, if adopted by all, cannot be destabilised by a single deviation (under a dynamic driven by imitation of more successful players).

The game $\bar{G}$ resembles a symmetric game because all players have the same strategy space, and any two players using the same strategy $\alpha^{i}=\alpha^{j}$ necessarily have the same payoff. But the game $\bar{G}$ is still not a symmetric game in the strict sense: if a large and a small investor with different strategies $\alpha^{i}, \alpha^{j}$ interchange their strategies, this may change prices and hence may change the other players' payoffs. Nevertheless, as argued above, we may define a concept of ESS in $\bar{G}$.

For a strategy $\alpha^{0} \in \Delta^{K}$, we denote by $\vec{\alpha}^{0}=\left(\alpha^{0}, \ldots \alpha^{0}\right) \in\left(\Delta^{K}\right)^{N}$ the symmetric profile in which every player uses $\alpha^{0}$. Let us call a strategy $\alpha^{0} \in \Delta^{K}$ an ESS of $\bar{G}$ if for every player $i$ and for every strategy $\alpha^{i} \in \Delta^{K}$ the following is true:

$$
\bar{\pi}^{i}\left(\left(\left.\alpha^{0}\right|_{i} \alpha^{i}\right)\right) \leq \bar{\pi}^{j}\left(\left(\left.\alpha^{0}\right|_{i} \alpha^{i}\right)\right) \quad \forall j \neq i
$$

where $\left(\left.\alpha^{0}\right|_{i} \alpha^{i}\right)$ denotes the strategy profile in which player $i$ uses strategy $\alpha^{i}$ and every other player uses the strategy $\alpha^{0}$. That is, if a player deviates from the symmetric profile $\vec{\alpha}^{0}$, then, after the deviation, 
his payoff is not larger than the payoff of any other player, so that nobody has an incentive to imitate the deviator. On the contrary, the deviator will have an incentive (at least in the weak inequality sense) to imitate one of the other players, i.e. to switch back to the ESS strategy $\alpha^{0}$.

Theorem 5.1. The game $\bar{G}$ has a unique ESS, namely $\alpha^{0}=\hat{\alpha}$, where $\hat{\alpha}$ is the competitive market portfolio corresponding to the "proportional competitive profile" $\hat{w}$ defined after Lemma 3.1. At this ESS, all players have the same payoff in $\bar{G}$, namely the competitive return $\bar{\pi}^{i}\left(\vec{\alpha}^{0}\right)=\hat{r} \forall i$.

Thus the ESS outcome is competitive, but different from the Nash outcome in general. Such a relation between ESS and competitive outcomes has been observed in other contexts as well, $c f$. [8,9].

\section{Acknowledgements}

I thank C. Alós - Ferrer, A. Ania, K. Podczeck and A. Ramsauer for helpful conversations.

\section{References}

1. Carlos, A.F.; Ania, Ana B. The asset market game. J. Math. Econ. 2005, 41, 67-90.

2. Blume, L.; Easley, D. Evolution and market behavior. J. Econ. Theor. 1992, 58, 9-40.

3. Hens, T.; Schenk-Hoppé, K.R. Evolutionary stability of portfolio rules in incomplete markets. J. Math. Econ. 2005, 41, 43-66.

4. Dubey, P.; Geanakoplos, J. From Nash to Walras via Shapley-Shubik. Cowles Foundation discussion paper 1360. J. Math. Econ. 2003, 39, 391-400

5. Hens, T.; Reimann, S.; Vogt, B. Nash competitive equilibria and two period fund separation. J. Math. Econ. 2004, 40, 321-346.

6. Shapley, L.; Shubik, M. Trade using one commodity as a means of payment. J. Polit. Econ. 1977, 85, 937-968.

7. Koutsougeras, L.C.; Papadopoulos, K.G. Arbitrage and equilibrium in strategic security markets. Econ. Theory 2004, 85, 553-568.

8. Carlos, A.F.; Ania, A.B. The evolutionary stability of perfectly competitive behavior. Econ. Theory 2005, 26, 497-516.

9. Vega-Redondo, F. The evolution of Walrasian Behavior. Econometrica 1997, 65, 375-384.

10. Ania, A.B. Evolutionary stability and Nash Equilibrium in finite populations, with an application to price competition. J. Econ. Behav. Org. 2008, 65, 472-488.

11. Schaffer, M.E. Evolutionarily stable strategies for a finite population and a variable contest size. J. Theor. Biol. 1988, 132, 469-478.

\section{A. Appendix}

\section{Lemma A.1. Under assumption S.1}

(i) for every $w_{k} \geq 0$, Equation (2) determines a unique price $p_{k}=P_{k}\left(w_{k}\right)$. The function $P_{k}\left(w_{k}\right)$ is continuous and strictly increasing on $[0, \infty)$, with

$$
P_{k}(0)=0, \quad \lim _{w_{k} \rightarrow \infty} P_{k}\left(w_{k}\right)=\infty
$$


(ii) The function $f_{k}\left(w_{k}\right):=w_{k} / P_{k}\left(w_{k}\right)$ is nondecreasing for $w_{k}>0$ and

$$
\lim _{w_{k} \rightarrow 0} w_{k} / P_{k}\left(w_{k}\right)=S_{k}(0)
$$

\section{Proof:}

(i) define the function

$$
V_{k}\left(p_{k}\right)=p_{k} S_{k}\left(p_{k}\right) \quad \text { for } p_{k} \geq 0
$$

By S.1, $V_{k}\left(p_{k}\right)$ is continuous and strictly increasing on $[0, \infty)$, with $V_{k}(0)=0$ and $\lim _{p_{k} \rightarrow \infty} V_{k}\left(p_{k}\right)=\infty$. Therefore $V_{k}$ has an inverse $V_{k}^{-1}$ with the same properties. Since equation (2) can be written $V_{k}\left(p_{k}\right)=$ $w_{k}$, the price function is equal to this inverse, $P_{k}\left(w_{k}\right)=V_{k}^{-1}\left(w_{k}\right)$.

(ii) For $w_{k}>0$, also $P_{k}\left(w_{k}\right)>0$, and $f_{k}\left(w_{k}\right)=w_{k} / P_{k}\left(w_{k}\right) \equiv S_{k}\left(P_{k}\left(w_{k}\right)\right)$. The assertion follows from $\mathbf{S . 1}$ and (i).

Remark: Conversely, the properties of the price function $P_{k}$ stated in Lemma A.1 imply that the supply function $S_{k}$ satisfies S.1. Indeed, if we postulate an arbitrary price function $P_{k}$ with the properties stated in Lemma A.1, and define a supply function $S_{k}$ by the condition $P_{k}\left(w_{k}\right) S_{k}\left(P_{k}\left(w_{k}\right)\right) \equiv w_{k}$ for $w_{k}>0$, and by (15) for $w_{k}=0$, then $S_{k}$ satisfies S.1. To see this, write $S_{k}\left(P_{k}\left(w_{k}\right)\right)=w_{k} / P_{k}\left(w_{k}\right)$ and observe that the 1-1-transformation $w_{k} \leftrightarrow p_{k}=P_{k}\left(w_{k}\right)$ is strictly increasing.

For future reference, we note that for any $c>0$

$$
S_{k}\left(P_{k}(\varepsilon)\right)>\varepsilon c \quad \forall \varepsilon>0 \text { sufficiently small }
$$

(Since $S_{k}\left(P_{k}(\varepsilon)\right) / \varepsilon=1 / P_{k}(\varepsilon)$ ).

The following is a more precise statement of the differentiability assumption in S.1. It is phrased so that an infinite slope at $p_{k}=0$ is not excluded.

S.4. For all $k$, the supply function $S_{k}\left(p_{k}\right)$ is either

(a) twice continuously differentiable on $[0, \infty)$ with $S_{k}^{\prime}(0)$ finite, or

(b) twice continuously differentiable on $(0, \infty)$, with

$$
\lim _{h \rightarrow 0}(S(h)-S(0)) / h=S_{k}^{\prime}(0)=\infty=\lim _{p_{k} \rightarrow 0} S_{k}^{\prime}\left(p_{k}\right)
$$

¿From now on, we maintain the assumptions S.1, S.4, S.3. Clearly, the elasticity function $\eta_{k}=H_{k}\left(p_{k}\right)=p_{k} S_{k}^{\prime}\left(p_{k}\right) / S_{k}\left(p_{k}\right)$ is continuously differentiable on $(0, \infty)$ and by $\mathbf{S . 3}$, the limit $\lim _{p_{k} \rightarrow 0} H_{k}\left(p_{k}\right)=: H_{k}(0) \in[0, \infty] \quad$ exists (possibly infinite).

Lemma A.2. There are only two possible cases: either

(i) supply is constant, $H_{k}(0)=0$, and $S_{k}\left(p_{k}\right)=S_{k}(0)=\bar{S}_{k}>0 \forall p_{k} \geq 0$, or

(ii) supply is not constant, $H_{k}(0)>0$, and $S_{k}\left(p_{k}\right)>S_{k}(0)=0 \forall p_{k}>0$; moreover

$(\alpha) S_{k}^{\prime}(0)=0$ if $H_{k}(0)>1$ (supply is elastic at 0 )

$(\beta) S_{k}^{\prime}(0)=\infty$ if $0<H_{k}(0)<1$ (supply is inelastic at 0 )

$(\gamma)$ if $H_{k}(0)=1$, it may be that $S_{k}^{\prime}(0)$ is positive and finite. (iii) in any case, $\lim _{p_{k} \rightarrow 0} p_{k} S_{k}^{\prime}\left(p_{k}\right)=0$ 
Proof: We omit the subscript $k$ for simplicity.

(i) Clearly, $H(0)=0$ iff $H(p)=0 \forall p>0$, i.e. iff $S^{\prime}(p)=0 \forall p>0$, i.e. iff supply is constant (and positive, by S.1): $S(p)=S(0)>0 \forall p \geq 0$. Obviously $(i i i)$ is satisfied in this case.

(ii) Assume now that supply is not constant. Then $H(0)>0$. By S.1 $S(p)>0$ for $p>0$. Next we show that $S(0)=0$. By definition,

$$
p S^{\prime}(p) \equiv H(p) S(p) \quad \forall p>0
$$

and

$$
\frac{d}{d p} \frac{S(p)}{p}=\frac{p S^{\prime}(p)-S(p)}{p^{2}} \quad \forall p>0
$$

Consider first the case of elastic supply at 0 , i.e. $H(0)>1$. Then, for $p>0$ sufficiently small, $H(p)>1$, and (18) implies: $p S^{\prime}(p)>S(p)$, i.e., by (19), the positive function $S(p) / p$ is strictly increasing in $p$. Therefore $\lim _{p \rightarrow 0}(S(p) / p)$ exists and is nonnegative and finite. This implies that $S(0)=0$, and furthermore that $S^{\prime}(0)=\lim _{p \rightarrow 0}(S(p)-S(0)) / p=\lim _{p \rightarrow 0} S(p) / p$ is finite. Moreover by (18):

$$
S^{\prime}(p) \equiv H(p) \frac{S(p)}{p}
$$

Both $S^{\prime}(p)$ and $S(p) / p$ tend to the same finite limit $S^{\prime}(0)$ as $p \rightarrow 0$, whereas $H(p)$ is bounded away from 1 for all $p$ sufficiently small. This is possible only if $S^{\prime}(0)=0$. This proves (ii)( $\alpha$ ). Clearly (iii) is also satisfied in this case.

Consider now the case of inelastic or unit elastic supply at $0,0<H(0) \leq 1$. Then, for $p>0$, $H(p) \leq 1$ by S.3, and (18) implies: $p S^{\prime}(p) \leq S(p)$, i.e., by (19), the positive function $S(p) / p$ is (weakly) decreasing in $p$. Therefore $\lim _{p \rightarrow 0}(S(p) / p$ ) exists and is strictly positive (possibly infinite).

Since

$$
H(p)=\frac{S^{\prime}(p)}{S(p) / p}
$$

is also weakly decreasing by $\mathbf{S . 3}$, the function $S^{\prime}(p)$ must be weakly decreasing, i.e. the supply function $S(p)$ is concave. This implies

$$
S^{\prime}(p) \leq \frac{S(p)-S(0)}{p} \quad \forall p>0
$$

It implies also that $S^{\prime}(0)>0$ (possibly $S^{\prime}(0)=\infty$ ), since otherwise $S^{\prime}(p) \equiv 0$ and supply would be constant.

We want to show that $S(0)=0$. If $S(0)>0$, then, for $p>0$ sufficiently small,

$$
S(p)\left[1-\frac{H(0)}{2}\right]<S(0)
$$

because $S($.$) is continuous and 0<H(0) \leq 1$. Therefore $S(p)-S(0)<\frac{1}{2} H(0) S(p)$ and, by (20)

$$
S^{\prime}(p)<\frac{H(0)}{2} \frac{S(p)}{p} \quad \text { for } p \text { sufficiently small. }
$$

On the other hand, by (18), $S^{\prime}(p) \equiv H(p) \frac{S(p)}{p}$ and for $p$ sufficiently small: $H(p)>\frac{1}{2} H(0)$ (because $H(0)>0$. This implies

$$
S^{\prime}(p)>\frac{H(0)}{2} \frac{S(p)}{p} \quad \text { for } p \text { sufficiently small, }
$$


contradicting (21). Therefore $S(0)=0$ for $0<H(0) \leq 1$ as well. Using (18) we see that

$$
\lim _{p \rightarrow 0} p S^{\prime}(p)=\lim _{p \rightarrow 0} H(p) S(p)=H(0) S(0)=0
$$

so that (iii) is also satisfied.

Finally, since $S(0)=0$, we have $S^{\prime}(0)=\lim _{p \rightarrow 0}(S(p) / p)$, and using (18) again:

$$
S^{\prime}(p)=H(p) \frac{S(p)}{p}
$$

If $p \rightarrow 0$, both $S^{\prime}(p)$ and $S(p) / p$ tend to the same positive limit $S^{\prime}(0)$ (possibly infinite) and $H(p)$ tends to $H(0)$. If $0<H(0)<1$ this is possible only if $S^{\prime}(0)=\infty$. This proves (ii) $(\beta)$. If $H(0)=1$, it is possible that $S^{\prime}(0)$ is positive and finite; e.g., for $S(p)=p, S^{\prime}(p)=1, H(p)=1 \quad \forall p \geq 0$. This proves (ii) $(\gamma)$ and the Lemma.

\section{Lemma A.3.}

(i) The function $V_{k}\left(p_{k}\right)=p_{k} S_{k}\left(p_{k}\right)$ is continuously differentiable on $[0, \infty)$, with $V_{k}^{\prime}\left(p_{k}\right)>0$ for $p_{k}>0$ and $V_{k}^{\prime}(0)=S_{k}(0)$.

(ii) The price function $P_{k}\left(w_{k}\right)$ is continuously differentiable on $[0, \infty)[$ resp. on $(0, \infty)]$, if $S_{k}(0)>0$ [resp. $S_{k}(0)=0$ ]; with $P_{k}^{\prime}\left(w_{k}\right)>0$ for $w_{k}>0$ and

$$
P_{k}^{\prime}(0)=\frac{1}{S_{k}(0)}=\lim _{w_{k} \rightarrow 0} P_{k}^{\prime}\left(w_{k}\right)
$$

(where $1 / S_{k}(0)=\infty$ if $\left.S_{k}(0)=0\right)$.

proof:

(i) For $p_{k}>0$, the assertions are trivial. At $p_{k}=0$, we have:

$$
V_{k}^{\prime}(0)=\lim _{\varepsilon \rightarrow 0}\left(V_{k}(\varepsilon)-V_{k}(0)\right) / \varepsilon=\lim _{\varepsilon \rightarrow 0}\left(\varepsilon S_{k}(\varepsilon)-0\right) / \varepsilon=S_{k}(0)
$$

For $p_{k}>0$, we have:

$$
V_{k}^{\prime}\left(p_{k}\right)=S_{k}\left(p_{k}\right)+p_{k} S_{k}^{\prime}\left(p_{k}\right)
$$

By Lemma A.2 (iii), the last term goes to zero for $p_{k} \rightarrow 0$, hence

$$
\lim _{p_{k} \rightarrow 0} V_{k}^{\prime}\left(p_{k}\right)=\lim _{p_{k} \rightarrow 0} S_{k}\left(p_{k}\right)=S_{k}(0)
$$

This proves (i).

(ii) The price function $P_{k}$ is the inverse of the function $V_{k}$. The assertions follow immediately from (i) and this fact, noting that $P_{k}^{\prime}\left(w_{k}\right)=1 / V_{k}^{\prime}\left(p_{k}\right)$ at all points where $V_{k}^{\prime}$ is positive, and that $P_{k}$ has infinite slope at zero iff $V_{k}^{\prime}(0)=0$.

Lemma A.4. The game $G$ is constant-sum (on the set $\left\{w \in B \mid w_{k}>0 \quad \forall k\right\}$ of strategies where all markets are active) if and only if all supply functions $S_{k}(\cdot)$ are constant.

Proof: The "if" part is trivial. Assume now that the game is constant-sum, i.e.,

$$
\sum_{i} \pi^{i}(w)=\sum_{i} \sum_{k} w_{k}^{i} R_{k}\left(w_{k}\right)=\sum_{k} w_{k} R_{k}\left(w_{k}\right)=\sum_{k} f_{k}\left(w_{k}\right)=\text { const. }
$$


for all $w_{k}>0$ with $\sum_{k} w_{k}=W$. This implies $f_{k}^{\prime}\left(w_{k}\right)=f_{\ell}^{\prime}\left(w_{\ell}\right)=c \forall w_{k}, w_{\ell}$, and $f_{k}\left(w_{k}\right)=c w_{k}+d_{k}$ $\forall k$, for some constants $c \geq 0, d_{k} \geq 0$. If $c>0$, then $S_{k}\left(P_{k}\left(w_{k}\right)\right)=f_{k}\left(w_{k}\right)=c w_{k}+d_{k}$ is not constant, hence $\lim _{p_{k} \rightarrow 0} S_{k}\left(p_{k}\right)=0$ by Lemma A.2, hence $d_{k}=\lim _{w_{k} \rightarrow 0} f_{k}\left(w_{k}\right)=0$. But then $f_{k}\left(w_{k}\right)=c w_{k}=$ $w_{k} R_{k}\left(w_{k}\right) \Rightarrow R_{k}\left(w_{k}\right)=c$, contradicting Lemma A.1. Therefore $c=0$ and $S_{k}\left[P_{k}\left(w_{k}\right)\right]=f_{k}\left(w_{k}\right)=$ $d_{k}>0$, i.e. supply $S_{k}$ is constant.

\section{Proof of Lemma 3.2.}

(i) Assume that all investors follow the proportional rule. Then

$$
w_{k}^{i}=\gamma^{i} E_{k} S_{k} \quad \forall i, k
$$

Summing this over $i$ gives $w_{k}=\left(\sum_{i} \gamma^{i}\right) E_{k} S_{k} \Leftrightarrow R_{k}=\left(E_{k} S_{k}\right) / w_{k}=\left(\sum_{i} \gamma^{i}\right)^{-1}=: \hat{r}$, i.e. the profile is competitive. Thus $\hat{w}_{k}$ is uniquely determined by $R_{k}\left(\hat{w}_{k}\right)=\hat{r}$. Hence $E_{k} S_{k}=\hat{r} \hat{w}_{k}$. Summing this over $k$ gives $E(w)=\hat{r} W$, and summing (23) over k gives $W^{i}=\gamma^{i} E(w) \quad \Leftrightarrow \quad \gamma^{i}=W^{i} / \hat{r} W$. Therefore $w_{k}^{i}=\gamma^{i} E_{k} S_{k}=\left(W^{i} / \hat{r} W\right) \hat{r} W=\hat{w}_{k} W / W^{i}$. This proves (i).

(ii) Since $w$ is competitive, $w_{k}=\hat{w}_{k}$ where $R_{k}\left(\hat{w}_{k}\right)=\hat{r} \forall k$. Since all agents hold the same portfolio $\alpha^{i}=\alpha^{j}$, summing $w_{k}^{i}=\alpha_{k}^{i} W^{i}$ over $i$ gives $\hat{w}_{k}=\alpha_{k}^{i} W \quad \Leftrightarrow \quad \alpha_{k}^{i}=\hat{w}_{k} / W \quad \Rightarrow \quad w_{k}^{i}=\alpha_{k}^{i} W^{i}=$ $\hat{w}_{k} W^{i} / W$. This is the allocation $\hat{w}$ given in part (i) of the lemma.

To prepare for the proof of Theorem 3.1, note that the payoff functions $\pi^{i}(w)$ are defined for all nonnegative vectors $w \in \mathbb{R}_{+}^{K N}$, independently of the agents' budget constraints. Clearly, the functions $x_{k}^{i}(w)$ and also the payoff functions $\pi^{i}(w)$ are differentiable in $w_{k}^{i}$ at all points where $w_{k}>0$ (with one-sided derivatives if $w_{k}^{i}=0$, but $w_{k}>0$ ). Denote by $\mathcal{W}^{a}=\left\{w \in \mathbb{R}_{+}^{K N} \mid w_{k}>0 \quad \forall k\right\}$ the set of profiles where all markets are active. Note that $\mathcal{W}^{a}$ is convex and the payoff functions $\pi^{i}(w)$ are continuous and differentiable on $\mathcal{W}^{a}$.

First we compute some derivatives. Let $w$ be a profile at which market $k$ is active, i.e., $w_{k}>0$, $p_{k}>0, R_{k}>0$. We have

$$
x_{k}^{i}(w)=\frac{w_{k}^{i}}{P_{k}\left(w_{k}\right)}=\frac{w_{k}^{i}}{w_{k}} S_{k}\left(P_{k}\left(w_{k}\right)\right)
$$

Therefore, from (12),

$$
\frac{\partial x_{k}^{i}(w)}{\partial w_{k}^{i}}=\frac{1}{P_{k}\left(w_{k}\right)}\left[1-\frac{w_{k}^{i}}{w_{k}} \frac{1}{1+H_{k}\left[P_{k}\left(w_{k}\right)\right]}\right] \geq 0
$$

with strict inequality unless investor $i$ is the only one who buys asset $k\left(w_{k}^{i}=w_{k}\right)$ and the supply elasticity is zero $\left(\eta_{k}=H_{k}\left[P_{k}\left(w_{k}\right)\right]=0\right)$. Also

$$
\frac{\partial x_{k}^{i}(w)}{\partial w_{k}^{i}} \leq \frac{1}{P_{k}}
$$

with strict inequality unless $w_{k}^{i}=0$, and

$$
\frac{\partial x_{k}^{i}(w)}{\partial w_{k}^{i}} \rightarrow \infty \text { for } w_{k} \rightarrow 0
$$

provided the expression $\left[1-\frac{w_{k}^{i}}{w_{k}} \frac{1}{1+\eta_{k}}\right]$ remains bounded away from 0 as $w_{k} \rightarrow 0$ (this is certainly the case if $w_{k}^{i} / w_{k}$ remains bounded away from 1 ). Moreover 


$$
\begin{aligned}
\frac{\partial^{2} x_{k}^{i}(w)}{\partial\left(w_{k}^{i}\right)^{2}}= & -\frac{P_{k}^{\prime}}{\left[P_{k}\right]^{2}}\left[1-\frac{w_{k}^{i}}{w_{k}} \frac{1}{1+H_{k}}\right]- \\
& -\frac{1}{P_{k}}\left[\frac{w_{k}-w_{k}^{i}}{\left(w_{k}\right)^{2}} \frac{1}{1+H_{k}}+\frac{w_{k}^{i}}{w_{k}} \frac{-H_{k}^{\prime} P_{k}^{\prime}}{\left(1+H_{k}\right)^{2}}\right] \leq 0
\end{aligned}
$$

where $P_{k}=P_{k}\left(w_{k}\right)$ and $H_{k}=H_{k}\left[P_{k}\left(w_{k}\right)\right]$. The inequality follows because $\eta_{k}^{\prime}=H_{k}^{\prime}\left(p_{k}\right) \leq 0$ by S.3, and is strict unless $w_{k}^{i}=w_{k}$ and both $\eta_{k}=0$ and $\eta_{k}^{\prime}=0^{10}$. The cross-partials are

$$
\frac{\partial^{2} x_{k}^{i}(w)}{\partial w_{k}^{i} \partial w_{\ell}^{i}}=0 \quad \text { for } \ell \neq k
$$

By (8) similar formulae hold for the profit functions $\pi^{i}$, e.g.,

$$
\frac{\partial \pi^{i}(w)}{\partial w_{k}^{i}}=E_{k} \frac{\partial x_{k}^{i}(w)}{\partial w_{k}^{i}} \geq 0 \text { for } w_{k}>0
$$

The formal proof of Theorem 3.1 is preceded by some lemmas.

Lemma A.5. For all $i$, the payoff function of investor $i, \pi^{i}(w)=\pi^{i}\left(w^{i}, w^{-i}\right)$ is concave in $i$ 's own strategy $w^{i}$ on the set $\mathcal{W}^{a}$, and even strictly concave except possibly at points where $w_{k}^{i}=w_{k}$ for some $k$ (investor $i$ is the only buyer of asset $k$ ).

Proof: We have, on the convex set $\mathcal{W}^{a}$ :

$$
\frac{\partial^{2} \pi^{i}(w)}{\partial\left(w_{k}^{i}\right)^{2}}=E_{k} \frac{\partial^{2} x_{k}^{i}(w)}{\partial\left(w_{k}^{i}\right)^{2}} \leq 0
$$

with strict inequality for $w_{k}^{i}<w_{k}$ and all cross-partials are zero.

Lemma A.6. Let $\bar{w}=\left(\bar{w}^{1}, \ldots \bar{w}^{N}\right) \in B$ be a strategy profile at which not all markets are active $\left(\bar{w} \notin \mathcal{W}^{a}\right)$. Then every investor has a profitable deviation, i.e. for every $i$ there exists a $\hat{w}^{i} \in B^{i}$ such that

$$
\pi^{i}\left(\hat{w}^{i}, \bar{w}^{-i}\right)>\pi^{i}(\bar{w})
$$

Moreover, $\hat{w}^{i}$ can be chosen so that at the new profile $\hat{w}=\left(\hat{w}^{i}, \bar{w}^{-i}\right)$ all markets are active.

Proof: Fix an investor $i$. Since he must invest his wealth somewhere, there exists an asset $m$ such that $\bar{w}_{m}^{i}>0\left(\Rightarrow \bar{w}_{m}>0, \bar{p}_{m}>0, \bar{R}_{m}>0\right)$. Let $\ell$ be an inactive asset so that $\bar{w}_{\ell}=\bar{w}_{\ell}^{i}=0$. Consider the following change in $i$ 's strategy, for small $\varepsilon>0$ :

$$
\hat{w}_{m}^{i}=\bar{w}_{m}^{i}-\varepsilon, \quad \hat{w}_{\ell}^{i}=\varepsilon, \quad \hat{w}_{k}^{i}=\bar{w}_{k}^{i} \quad \text { for } k \neq m, \ell .
$$

That is, investor $i$ shifts a small amount $\varepsilon$ from asset $m$ to the inactive asset $\ell$. This shift decreases his earnings in market $m$ by (using (26), (30))

$$
0 \leq \varepsilon \frac{\partial \pi^{i}(\bar{w})}{\partial w_{m}^{i}} \leq \varepsilon E_{m} \frac{1}{\bar{p}_{m}}=\varepsilon \bar{R}_{m}
$$

${ }^{10} \eta^{\prime}=0$ is implied by $\eta=0$ because $\eta \geq 0$ and nondecreasing. 
and it increases his earnings in market $\ell$ by $E_{\ell} S_{\ell}\left(P_{\ell}(\varepsilon)\right)$. By (17) this is strictly greater than $\varepsilon \bar{R}_{m}$ for $\varepsilon$ sufficiently small, i.e. (32) is satisfied. If $\ell$ is the only inactive market at $\bar{w}$, we are done. If not, repeat the construction for the next inactive asset, starting from the profile $\hat{w}=\left(\hat{w}^{i}, \bar{w}^{-i}\right)$.

As an immediate Corollary we have that all markets must be active at equilibrium.

Lemma A.7. If $w$ is an equilibrium, then

$$
\frac{\partial \pi^{i}(w)}{\partial\left(w_{k}^{i}\right)}>0 \quad \text { and } \quad \frac{\partial^{2} \pi^{i}(w)}{\partial\left(w_{k}^{i}\right)^{2}}<0
$$

for all $i=1, \ldots N, k=1, \ldots K$.

Proof: By Lemma A.6, $w_{k}>0$ at equilibrium. Therefore, by (25), (28), both claims are true unless

$$
w_{k}^{i}=w_{k} \quad \text { and } \quad \eta_{k}=0 \quad\left(\text { and } \quad \eta_{k}^{\prime}=0\right)
$$

We have to show that this situation is impossible at equilibrium.

Indeed, if (34) holds, then $w_{k}^{i}=w_{k}>0$ and $\partial \pi^{i}(w) / \partial w_{k}^{i}=0$ by (25).

On the other hand. there must exist an asset $\ell$ with $w_{\ell}^{i}<w_{\ell}$, hence by (25)

$$
\partial \pi^{i}(w) / \partial w_{\ell}^{i}>0
$$

Shifting a small amount $\varepsilon>0$ from asset $k$ to asset $\ell$ increases $i$ 's profits, contradicting equilibrium.

In particular, if an asset is in constant supply, then it must be held by more than one investor at equilibrium. Moreover, if $w^{*}=\left(w^{* i}, w^{*-i}\right)$ is an equilibrium, then the payoff function $\pi\left(w^{i}, w^{*-i}\right)$ is strictly concave in $w^{i}$ in a neighborhood of $w^{* i}$, and concave elsewhere. Therefore every equilibrium is strict.

\section{Proof of Theorem 3.1.}

Assertions (ii) and (iii) follow from the two preceding Lemmas. It only remains to prove assertion (i) (existence of Nash equilibrium).

For $\nu=1,2,3, \ldots$ consider the modified game $G^{\nu}$ with budget sets $B^{i}(\nu)=\left\{w^{i} \in B^{i} \mid w_{k}^{i} \geq\right.$ $\left.\frac{1}{\nu} \forall k\right\}$ and payoff functions $\pi^{i}$ as before. Eventually, for $\nu$ sufficiently large, $B^{i}(\nu)$ is nonempty, compact, convex. Clearly at any $w \in B(\nu)=\prod_{i} B^{i}(\nu) \subset \mathcal{W}^{a}$ all markets are active and each payoff function $\pi^{i}(w)=\pi^{i}\left(w^{i}, w^{-i}\right)$ is continuous in $w \in B(\nu)$ and strictly concave in the own strategy $w^{i}$. Therefore there exists an equilibrium $w(\nu)=\left(w^{1}(\nu), \ldots w^{N}(\nu)\right)$ of the modified game $G^{\nu}$, where of course $w_{k}^{i}(\nu) \geq \frac{1}{\nu}$ always.

W.l.o.g. (passing to a subsequence if necessary) we may assume that the sequence $w(\nu)_{\nu=1,2, \ldots}$ converges, i.e.,

$$
w(\nu) \rightarrow w^{*}=\left(w^{* 1}, \ldots w^{* N}\right) \quad \text { for } \nu \rightarrow \infty
$$

We claim that $w^{*}$ is an equilibrium in the unrestricted game $G$ with strategy spaces $B^{i}$. We proceed in two steps:

Step 1. $w_{k}^{*}>0 \quad \forall k$, i.e. $w^{*} \in \mathcal{W}^{a}$

Step 2. $\forall i, w^{* i}$ is a best reply to $w^{*}$ 
Step 1.

Assume, indirectly, that there is an asset $\ell$ with $w_{\ell}^{*}=0$, i.e., $w_{\ell}(\nu) \rightarrow 0$ for $\nu \rightarrow \infty$. Of course then also $w_{\ell}^{i}(\nu) \rightarrow 0$ for each agent $i$; but since $\sum_{i} w_{\ell}^{i}(\nu)=w_{\ell}(\nu)>0$ always, there exists an agent $j$ such that $w_{\ell}^{j}(\nu) / w_{\ell}(\nu)$ remains bounded away from 1 as $\nu \rightarrow \infty$ (taking a further subsequence if necessary). By (27) this implies

$$
\frac{\partial x_{\ell}^{j}(w(\nu))}{\partial w_{\ell}^{j}} \rightarrow \infty \quad \text { as } \nu \rightarrow \infty
$$

There must also exist some asset $m \neq \ell$ such that $w_{m}^{* j}>0$, and hence $w_{m}^{*}>0$. Fix $j, \ell, m$. Then

$$
\frac{\partial x_{m}^{j}(w(\nu))}{\partial w_{m}^{j}}=\frac{1}{P_{m}\left(w_{m}(\nu)\right)}\left[1-\frac{w_{m}^{j}(\nu)}{w_{m}(\nu)} \frac{1}{1+H_{m}\left(P_{m}\left(w_{m}(\nu)\right)\right)}\right]
$$

converges to the finite number

$$
\frac{1}{P_{m}\left(w_{m}^{*}\right]}\left[1-\frac{w_{m}^{* j}}{w_{m}^{*}} \frac{1}{1+H_{m}\left(P_{m}\left(w_{m}^{*}\right)\right)}\right]=: c_{m} \geq 0
$$

as $\nu \rightarrow \infty$. Therefore, for $\nu$ sufficiently large, agent $j$ can increase his payoff $\pi^{j}(w(\nu))$ in the game $G^{\nu}$ by shifting a small amount $\varepsilon>0$ away from asset $m$ (this is feasible because eventually $w_{m}^{* j}>\frac{1}{\nu}$ ) to asset $\ell$. This contradicts the assumption that $w(\nu)$ is an equilibrium in $G^{\nu}$ and proves Step 1 .

Step 2.

By Step $1, \pi^{i}(w)$ is continuous at $w^{*}$. Fix an investor $i$. We have to show that $w^{* i}$ is a best reply to $w^{*-i}$. Assume not. Then there exists $\hat{w}^{i} \in B^{i}$ which is a better reply to $w^{*-i}$, i.e.

$$
\pi^{i}\left(\hat{w}^{i}, w^{*-i}\right)-\pi^{i}\left(w^{*}\right)>\delta \quad \text { for some } \delta>0
$$

If the strategy profile $\left(\hat{w}^{i}, w^{*-i}\right) \notin \mathcal{W}^{a}$, then by Lemma A.6 there exists a further deviation $\hat{\hat{w}}^{i}$ such that $\left(\hat{\hat{w}}^{i}, w^{*-i}\right) \in \mathcal{W}^{a}$ and $\pi^{i}\left(\hat{\hat{w}}^{i}, w^{*-i}\right)>\pi^{i}\left(\hat{w}^{i}, w^{*-i}\right)$. Therefore we may assume that (35) holds with $\hat{w}:=\left(\hat{w}^{i}, w^{*-i}\right) \in \mathcal{W}^{a}$, so that $\pi^{i}(\cdot)$ is continuous at this point. Approximate $\hat{w}^{i} \in B^{i}$ by a sequence $\hat{w}^{i}(\nu) \in B^{i}(\nu)$. Then by continuity

$$
\pi^{i}\left(\hat{w}^{i}(\nu), w^{-i}(\nu)\right)-\pi^{i}(w(\nu))>\frac{\delta}{2}>0
$$

for $\nu$ sufficiently large, i.e. $w(\nu)$ is not an equilibrium of $G^{\nu}$, contrary to assumption. This proves Step 2 and completes the proof of Theorem 3.1.

\section{Proof of Theorem 4.1.}

Consider a Nash equilibrium $w=\left(w^{1}, \ldots w^{N}\right)$ with associated prices $p_{k}$, asset returns $r_{k}$, and elasticities $\eta_{k}$. Denote by $\alpha^{i}=\left(\alpha_{1}^{i}, \ldots \alpha_{K}^{i}\right)=\left(1 / W^{i}\right) w^{i}$ the equilibrium portfolio, and by $r^{i}:=\pi^{i}(w) / W^{i}$ the average return of investor $i$.

The following proof is based on a careful examination of the first-order conditions for a Nash equilibrium. To understand the following arguments, it helps to keep Table 1 in mind.

Proof of Assertions 4.1-2:

Let $w$ be a Nash equilibrium. By (13)

$$
\frac{\partial \pi^{i}(w)}{\partial w_{k}^{i}}=r_{k}\left[1-\frac{w_{k}^{i}}{w_{k}} \frac{1}{1+\eta_{k}}\right]
$$


Denote by $\lambda^{i}$ the Lagrange multiplier associated with investor $i$ 's budget constraint. Then the following first-order conditions [FOC] must hold, for $i=1, \ldots N$ :

$$
\frac{\partial \pi^{i}(w)}{\partial w_{k}^{i}}= \begin{cases}r_{k}\left[1-\frac{w_{k}^{i}}{w_{k}} \frac{1}{1+\eta_{k}}\right] & =\lambda^{i} \quad \forall k \text { with } w_{k}^{i}>0 \\ r_{k} & \leq \lambda^{i} \quad \forall k \text { with } w_{k}^{i}=0\end{cases}
$$

By Lemma A.7, $\lambda^{i}>0$ for $\forall i$. (13) implies, $\forall i, \forall k$ :

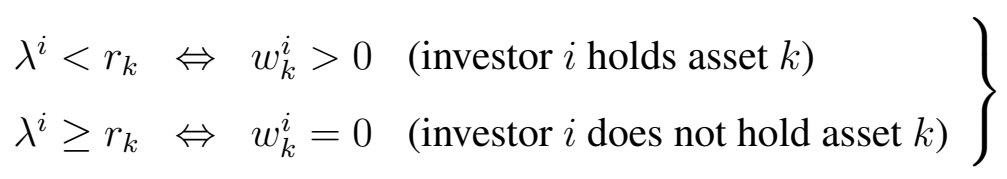

W.l.o.g., order the investors such that

$$
\lambda^{1} \leq \lambda^{2} \leq \cdots \leq \lambda^{N}
$$

and order the assets such that

$$
r_{1} \leq r_{2} \leq \cdots \leq r_{K}
$$

We shall see below (see (46)) that (39) implies $W^{1} \geq W^{2} \geq \ldots W^{N}$, i.e., investor $i=1$ is the largest and $i=N$ is the smallest investor. Similarly, asset $k=1$ is the worst and $k=K$ is the best asset, where "better" assets have higher returns per dollar invested.

For given $i$, define

$$
k^{i}:=\min \left\{k \mid \lambda^{i}<r_{k}\right\} \quad \text { (worst asset held by } i \text { ) }
$$

and for given $k$, define

$$
i_{k}:=\max \left\{i \mid \lambda^{i}<r_{k}\right\} \quad \text { (smallest investor holding } k \text { ) }
$$

It is easy to see that investor $i$ holds exactly the assets $k=k^{i}, k^{i}+1, \ldots K$ and

$$
1=k^{1} \leq k^{2} \leq \cdots \leq k^{N} \leq K
$$

with $k^{i}=k^{j}$ if $\lambda^{i}=\lambda^{j}$. Similarly, it is also easy to see that asset $k$ is held exactly by the investors $i=1,2, \ldots i_{k}$ and

$$
1 \leq i_{1} \leq i_{2} \leq \cdots \leq i_{K}=N
$$

with $i_{k}=i_{\ell}$ if $r_{k}=r_{\ell}$.

The equilibrium allocation $w=\left(w_{k}^{i}\right)$ is summarized in Table 1 .

The largest investor $i=1$ holds all assets $\left(w_{k}^{1}>0 \forall k\right)$ and the best asset $k=K$ is held by all investors $\left(w_{K}^{i}>0 \forall i\right)$. For fixed $k$, we know from (13), (39) that

$$
w_{k}^{i} \geq w_{k}^{i+1}
$$

with strict inequality iff $\left[w_{k}^{i}>0\right.$ and $\left.\lambda^{i}<\lambda^{i+1}\right]$. Therefore the row sums $W^{i}$ in Table 1 satisfy

$$
W^{1} \geq W^{2} \geq \cdots \geq W^{N}
$$


with strict inequality

$$
W^{i}>W^{i+1} \quad \text { iff } \lambda^{i}<\lambda^{i+1}
$$

(since $w_{K}^{i}>0 \forall i$ ). This proves the first three assertions in Theorem 4.1.

\section{Proof of assertion 5:}

let $r_{k} \leq r_{\ell}$. Then $i_{k} \leq i_{\ell}$ and w.l.o.g. $k<\ell$. Summing the first line in (13) for asset $k$ over $i=1, \ldots i_{k}$ gives (since $\sum_{i=1}^{i_{k}} w_{k}^{i}=w_{k}$ )

$$
r_{k}\left[i_{k}-\frac{1}{1+\eta_{k}}\right]=\sum_{i=1}^{i_{k}} \lambda^{i}=: \Lambda
$$

Summing the first line in (13) for asset $\ell$ also over $i=1, \ldots i_{k}$ gives

$$
\begin{gathered}
r_{\ell}\left[i_{k}-\frac{1}{1+\eta_{\ell}}\right]=\Lambda \quad \text { if } i_{\ell}=i_{k} \\
r_{\ell}\left[i_{k}-\frac{\sum_{i=1}^{i_{k}} w_{\ell}^{i}}{w_{\ell}} \frac{1}{1+\eta_{\ell}}\right]=\Lambda \quad \text { if } i_{\ell}>i_{k}
\end{gathered}
$$

If $i_{\ell}=i_{k}$, the assertion follows directly from (48), (49). If $i_{\ell}>i_{k}$, then necessarily $r_{\ell}>r_{k}$ (per def. of $i_{k}$ ), and (48), (50) imply

$\Rightarrow$

$$
i_{k}-\frac{1}{1+\eta_{k}}>i_{k}-\frac{\sum_{i=1}^{i_{k}} w_{\ell}^{i}}{w_{\ell}} \frac{1}{1+\eta_{\ell}}
$$

$$
\frac{1}{1+\eta_{k}}<\frac{\sum_{i=1}^{i_{k}} w_{\ell}^{i}}{w_{\ell}} \frac{1}{1+\eta_{\ell}} \leq \frac{1}{1+\eta_{\ell}}
$$

$\Rightarrow \eta_{k}>\eta_{\ell}$, and assertion 5 is proved.

\section{Proof of assertion 6:}

write $r^{i}=\pi^{i}(w) / W^{i}=\sum_{k}\left(w_{k}^{i} / W^{i}\right) r_{k}=\sum_{k} \alpha_{k}^{i} r_{k}$, where $\alpha_{k}^{i}=w_{k}^{i} / W^{i}$ is the portfolio associated with the equilibrium strategy $w^{i}$. By Abel's summation formula, we can write

$$
r^{i}=\sum_{k=1}^{K} \alpha_{k}^{i} r_{k}=A_{K}^{i} r_{K+1}+\sum_{k=1}^{K} A_{k}^{i}\left(r_{k}-r_{k+1}\right)
$$

where $A_{k}^{i}=\sum_{\ell=1}^{k} \alpha_{\ell}^{i}$ and $r_{K+1}$ is arbitrary.

Now fix two investors $i \geq j$ so that $W^{i} \leq W^{j}$ (investor $i$ is smaller). We want to show that $r^{i} \geq r^{j}$. From Abel's formula, noting that $A_{K}^{i}=A_{K}^{j}=1$ :

$$
r^{i}-r^{j}=\sum_{k=1}^{K-1}\left(A_{k}^{i}-A_{k}^{j}\right)\left(r_{k}-r_{k+1}\right)
$$

Since $r_{k} \leq r_{k+1}$ it suffices to show that $A_{k}^{i} \leq A_{k}^{j}$ for $k=1, \ldots K-1$, or equivalently,

$$
\sum_{\ell=k+1}^{K} \alpha_{\ell}^{i} \geq \sum_{\ell=k+1}^{K} \alpha_{\ell}^{j} \quad \text { for } \quad k=1, \ldots K-1
$$

(because $A_{\ell}^{i}=1-\sum_{\ell=k+1}^{K} \alpha_{\ell}^{i}$ ). Thus assertion 6 follows from assertion 4 . 


\section{Proof of assertion 4:}

Note that the coefficients $\alpha_{k}^{i}$ have also the "triangular structure" exhibited in Table 1: If some $\alpha_{k}^{i}>0$, then also $\alpha_{k+1}^{i}>0, \alpha_{k}^{j}>0, \alpha_{k+1}^{j}>0$; and $r_{k}-\lambda^{i}>0$.

For $\alpha_{k}^{i}>0$ the first-order condition (13) can be written

$$
\begin{gathered}
r_{k}\left[1-\alpha_{k}^{i} \frac{W^{i}}{w_{k}} \frac{1}{1+\eta_{k}}\right]=\lambda^{i} \Leftrightarrow \\
\alpha_{k}^{i}=\frac{r_{k}-\lambda^{i}}{W^{i}} \frac{w_{k}\left(1+\eta_{k}\right)}{r_{k}}
\end{gathered}
$$

This implies

$$
\frac{\alpha_{k}^{i}}{\alpha_{k+1}^{i}}=\frac{\frac{w_{k}\left(1+\eta_{k}\right)}{r_{k}} \frac{r_{k}-\lambda^{i}}{W^{i}}}{\frac{w_{k+1}\left(1+\eta_{k+1}\right)}{r_{k+1}} \frac{r_{k+1}-\lambda^{i}}{W^{i}}}
$$

and

$$
\frac{\alpha_{k}^{i} / \alpha_{k+1}^{i}}{\alpha_{k}^{j} / \alpha_{k+1}^{j}}=\frac{\left(r_{k}-\lambda^{i}\right) /\left(r_{k+1}-\lambda^{i}\right)}{\left(r_{k}-\lambda^{j}\right) /\left(r_{k+1}-\lambda^{j}\right)}=: B
$$

(all quantities are positive by the remark made above). From Table 1 we know that $\lambda^{i} \geq \lambda^{j}$, so that $r_{k+1} \geq r_{k}>\lambda^{i} \geq \lambda^{j}$. But then the function $f(\lambda)=\left(r_{k}-\lambda\right) /\left(r_{k+1}-\lambda\right)$ is decreasing in $\lambda$, hence $B \leq 1$. This implies

$$
\frac{\alpha_{k}^{i}}{\alpha_{k+1}^{i}} \leq \frac{\alpha_{k}^{j}}{\alpha_{k+1}^{j}}
$$

It remains to show that this implies (51).

Let $k_{0}$ be the first (smallest) $k$ such that $\alpha_{k}^{i}>0$. Then for $k_{0} \leq k \leq m \leq K$

$$
\alpha_{k}^{i}=\beta_{k m}^{i} \alpha_{m}^{i}
$$

where

$$
\beta_{k m}^{i}:=\frac{\alpha_{k}^{i}}{\alpha_{k+1}^{i}} \frac{\alpha_{k+1}^{i}}{\alpha_{k+2}^{i}} \cdots \frac{\alpha_{m-1}^{i}}{\alpha_{m}^{i}} \quad \text { for } \quad k<m \quad \text { and } \quad \beta_{m m}^{i}=1
$$

Defining $\beta_{k m}^{j}$ similarly, we see from (52) that $\beta_{k m}^{i} \leq \beta_{k m}^{j}$ for $k_{0} \leq k \leq m \leq K$.

Claim 1: $\alpha_{K}^{i} \geq \alpha_{K}^{j}$

Proof: assume the contrary, $\alpha_{K}^{i}<\alpha_{K}^{j}$. Then $\alpha_{k}^{i}=\beta_{k K}^{i} \alpha_{K}^{i}<\beta_{k K}^{j} \alpha_{K}^{j}=\alpha_{k}^{j}$ for $k_{0} \leq k \leq K-1$, and $\sum_{k=1}^{K} \alpha_{k}^{i}=\sum_{k=k_{0}}^{K} \alpha_{k}^{i}<\sum_{k=1}^{K} \alpha_{k}^{j}$. But this is impossible because both sums must be equal to one.

Claim 2: $\alpha_{K}^{i}+\alpha_{K-1}^{i} \geq \alpha_{K}^{j}+\alpha_{K-1}^{j}$

Proof: assume the contrary, $\alpha_{K}^{i}+\alpha_{K-1}^{i}<\alpha_{K}^{j}+\alpha_{K-1}^{j}$. Then $\alpha_{K-1}^{i}<\alpha_{K-1}^{j}$ by Claim 1. Therefore $\alpha_{k}^{i}=\beta_{k, K-1}^{i} \alpha_{K-1}^{i}<\beta_{k, K-1}^{j} \alpha_{K-1}^{j}=\alpha_{k}^{j}$ for $k_{0} \leq k \leq K-2$. Again the same contradiction arises.

Claim 3: $\alpha_{K}^{i}+\alpha_{K-1}^{i}+\alpha_{K-2}^{i} \geq \alpha_{K}^{j}+\alpha_{K-1}^{j}+\alpha_{K-2}^{j}$

Proof: as before, assuming the contrary implies $\alpha_{K-2}^{i}<\alpha_{K-2}^{j}$ by Claim 2, and this implies that $\alpha_{k}^{i}<\alpha_{k}^{j}$ for $k_{0} \leq k \leq K-3$, leading to a contradiction.

Proceeding in this manner until $K-\ell=k_{0}$, we obtain all the inequalities (51) (the remaining ones are trivial). This proves assertion 4 and completes the proof of Theorem 4.1. 


\section{Proof of Proposition 4.2.}

Let $w$ be an equilibrium, with $w_{k}^{i}>0 \forall i, \forall k$. Then the first-order conditions (13) take the form (remember $r_{k}=E_{k} / p_{k}$ )

$$
E_{k}\left[1-\frac{w_{k}^{i}}{w_{k}} \frac{1}{1+\eta_{k}}\right]=\lambda^{i} p_{k} \quad \forall i, k
$$

Summing over $i$ gives

$$
E_{k}\left[N-\frac{1}{1+\eta_{k}}\right]=\Lambda p_{k} \quad \forall k
$$

where $\Lambda:=\sum_{i} \lambda^{i}>0$. The RHS of this equation is strictly increasing in $p_{k}$, and the LHS is weakly decreasing in $p_{k}$ by S.3, hence, for any given $\Lambda$, there exists only one solution $p_{k}$. If $\Lambda$ increases, the curve described by the RHS shifts upwards, and the LHS does not change, i.e., the intersection point with the LHS shifts to the left, i.e., $p_{k}$ decreases, $\forall k$. Since the price function $p_{k}=P_{k}\left(w_{k}\right)$ is strictly increasing, $w_{k}=P_{k}^{-1}\left(p_{k}\right)$ also decreases strictly in $\Lambda, \forall k$. Since $\sum_{k} w_{k}=\sum_{i} W^{i}=W$ is constant, the numbers $w_{k}$ (and hence also $p_{k}, \eta_{k}$ ) are uniquely determined by (54) and the condition $\sum_{k} w_{k}=W$. Given this, the numbers $w_{k}^{i}$ (and the multipliers $\lambda^{i}$ ) are uniquely determined by (53) and the budget constraints $\sum_{k} w_{k}^{i}=W^{i}$.

\section{Proof of Theorem 4.4.}

Write $r_{k}^{*}=R_{k}\left(w_{k}^{*}\right)$ for the Nash quantities and $\hat{r}=R_{k}\left(\hat{w}_{k}\right)$ for the competitive values. Clearly,

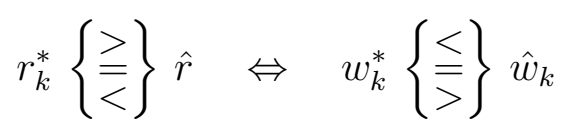

Fix an investor $i$. Denote by $w_{k}^{*-i}=w_{k}^{*}-w_{k}^{* i}$ the total amount of money invested in asset $k$ by the 'others'. Define the sets $K_{0}=\left\{k \mid \hat{w}_{k}>w_{k}^{*-i}\right\}, K_{1}=\left\{k \mid \hat{w}_{k}=w_{k}^{*-i}\right\}, K_{2}=\left\{k \mid \hat{w}_{k}<w_{k}^{*-i}\right\}$. Then $K_{0}$ is nonempty (because $\sum_{k} \hat{w}_{k}=W>W-W^{i}=\sum_{k} w_{k}^{*-i}$ ), and

$$
W^{i}=W-\sum_{k} w_{k}^{*-i}=\sum_{k}\left(\hat{w}_{k}-w^{*-i}\right)=\sum_{k \in K_{0}}\left(\hat{w}_{k}-w^{*-i}\right)+\sum_{k \in K_{2}}\left(\hat{w}_{k}-w^{*-i}\right)
$$

If $K_{2} \neq \emptyset$, the last sum is negative, hence $\sum_{k \in K_{0}}\left(\hat{w}_{k}-w^{*-i}\right)>W^{i}$. Therefore, investor $i$ can find a deviating strategy $w^{i} \in B^{i}$ such that $w_{k}^{i}=0 \forall k \notin K_{0}$ and $\hat{w}_{k}>w_{k}^{*-i}+w_{k}^{i} \forall k \in K_{0}$. Then $R_{k}\left(w_{k}^{*-i}+w_{k}^{i}\right)>\hat{r}$ for $k \in K_{0}$ and $\pi^{i}\left(w^{i}, w^{*-i}\right)=\sum_{k \in K_{0}} w_{k}^{i} R_{k}\left(w_{k}^{*-i}+w_{k}^{i}\right)>W^{i} \hat{r}$, i.e. with the strategy $w^{i}$ the investor achieves a rate of return higher than $\hat{r}$. Since $w^{* i}$ is a best reply to $w^{*-i}$, we have $\pi^{i}\left(w^{*}\right) \geq \pi^{i}\left(w^{i}, w^{*-i}\right)>\hat{r}$.

If $K_{2}=\emptyset$, then $w_{k}^{*-i} \leq \hat{w}_{k} \forall k$ and the strategy $w^{i}$ given by $w_{k}^{i}:=\hat{w}_{k}-w_{k}^{*-i} \forall k$ is feasible for investor $i$. The profile $\left(w^{i}, w^{*-i}\right) \in B$ is competitive and guarantees player $i$ the return $\hat{r}$. By assumption, the equilibrium $w^{*}$ is not competitive, hence $w^{* i} \neq w^{i}$. Moreover, since any equilibrium is strict by Theorem 3.1, we must have $\pi^{i}\left(w^{*}\right)>\pi^{i}\left(w^{i}, w^{*-1}\right)=\hat{r}$.

\section{Proof of Theorem 5.1.}

By Lemma 3.2 (ii) there exists a unique, strictly positive $\alpha^{0}=\left(\alpha_{1}^{0}, \ldots \alpha_{K}^{0}\right) \in \Delta^{K}$ such that the return $r_{k}=R_{0}=\hat{r}$ in all markets is the same, viz. $\alpha^{0}=\hat{\alpha}$. We claim that this $\hat{\alpha}$ is ESS.

Fix an investor $i$ and let him deviate to some strategy $\alpha^{0}+\varepsilon \in \Delta^{K}$, where $\varepsilon=\left(\varepsilon_{1}, \ldots \varepsilon_{K}\right) \neq 0$ and of course $\sum_{k} \varepsilon_{k}=0$. Denote by $\alpha^{\prime}$ the new profile where player $i$ uses strategy $\alpha^{0}+\varepsilon$ and all other players use strategy $\alpha^{0}$. Write $R_{0}+d R_{k}$ for the return rate in market $k$ after this deviation, i.e., at the profile $\alpha^{\prime}$. 
The crucial observation is that $\forall k \quad \varepsilon_{k} d R_{k}<0$ if $\varepsilon_{k} \neq 0$. Indeed, if $\varepsilon_{k}>0$, then more money is invested in asset $k$, which strictly increases the price of this asset and strictly decreases the return $R_{k}$; and conversely for $\varepsilon_{k}<0^{11}$.

The deviator's payoff after the deviation is

$$
\bar{\pi}^{i}\left(\alpha^{\prime}\right)=\sum_{k}\left(\alpha_{k}^{0}+\varepsilon_{k}\right)\left(R_{0}+d R_{k}\right)=\sum_{k} \alpha_{k}^{0}\left(R_{0}+d R_{k}\right)+\sum_{k} \varepsilon_{k} R_{0}+\sum_{k} \varepsilon_{k} d R_{k}
$$

The second term in the last expression is zero, and the third term is negative, by the observations made above. Therefore the payoff of a non-deviator $j(j \neq i)$ after the deviation is greater:

$$
\bar{\pi}^{j}\left(\alpha^{\prime}\right)=\sum_{k} \alpha_{k}^{0}\left(R_{0}+d R_{k}\right)>\bar{\pi}^{i}\left(\alpha^{\prime}\right)
$$

This proves that $\alpha^{0}$ is an ESS. It remains to show uniqueness.

Let $\beta^{0} \neq \alpha^{0}$ be any other strategy. We have to show that the symmetric profile $\vec{\beta}^{0}=\left(\beta^{0}, \ldots \beta^{0}\right) \in$ $\left(\Delta^{K}\right)^{N}$ is not an ESS, i.e., we have to show that there exists a deviation $\varepsilon=\left(\varepsilon_{1}, \ldots \varepsilon_{K}\right)$ with $\sum_{k} \varepsilon_{k}=0$ which improves the deviator's relative position.

First it is clear that if $\beta_{k}^{0}=0$ for some $k$, then the rate of return for sufficiently small investments in market $k$ is arbitrarily large, and a small shift of money into market $k$ helps the deviator more than the others. Assume therefore that $\beta^{0}$ is strictly positive.

Denote by $R_{k}$ the rate of return in market $k$ under the symmetric profile $\vec{\beta}^{0}$. Since $\beta^{0} \neq \alpha^{0}$, we know from the first part of the proof that not all $R_{k}$ are equal, i.e. the vector $\left(R_{1}, \ldots R_{K}\right)$ is not orthogonal to the hyperplane $L=\left\{z=\left(z_{1}, \ldots z_{K}\right) \in \mathbb{R}^{K} \mid \sum_{k} z_{k}=0\right\}$ in $\mathbb{R}^{K}$. Therefore we can find a vector $z \in L$ such that $\sum_{k} z_{k} R_{k}>0$. Clearly, for $t>0$ sufficiently small, the vector $\varepsilon=\varepsilon(t)=t z$ is a feasible deviation, i.e. $\beta^{0}+\varepsilon(t) \in \Delta^{K}$.

Denote by $R_{k}+d R_{k}$ the return rate in market $k$ after such a deviation, i.e. at the new profile $\beta^{\prime}=\left(\left.\beta^{0}\right|_{i} \beta^{0}+\varepsilon\right)$. Then, for the deviator $i$ and any non-deviator $j$ :

$$
\begin{array}{r}
\bar{\pi}^{i}\left(\beta^{\prime}\right)-\bar{\pi}^{j}\left(\beta^{\prime}\right)=\sum_{k}\left(\beta_{k}^{0}+\varepsilon_{k}\right)\left(R_{k}+d R_{k}\right)-\sum_{k} \beta_{k}^{0}\left(R_{k}+d R_{k}\right)= \\
\sum_{k} \varepsilon_{k}\left(R_{k}+d R_{k}\right)=\sum_{k} t z_{k}\left(R_{k}+d R_{k}\right)=t \sum_{k} z_{k}\left(R_{k}+d R_{k}\right)
\end{array}
$$

The last term is positive for $t>0$ sufficiently small, because $d R_{k} \rightarrow 0$ for $t \rightarrow 0$. Thus we have found a deviation which makes the deviator better off than the others, and $\beta^{0}$ is not an ESS.

(c) 2011 by the author; licensee MDPI, Basel, Switzerland. This article is an open access article distributed under the terms and conditions of the Creative Commons Attribution license (http://creativecommons.org/licenses/by/3.0/.)

\footnotetext{
${ }^{11}$ This argument parallels the one in [1]. Intuitively, as also pointed out in this paper, if an investor shifts money from one market to another, he "works against himself" by increasing the price of the asset which he is buying, and decreasing the price of the asset which he is selling.
} 